\title{
Highly Regio- and Stereoselective Rearrangement of Epoxides to Aldehydes Catalyzed by High-Valent Metalloporphyrin Complex, Cr(TPP)OTf
}

\author{
Kohji Suda,* Taketoshi Kikkawa, Shin-ichiro Nakajima, and Toshikatsu Takanami \\ Meiji Pharmaceutical University, 2-522-1, Noshio, Kiyose, Tokyo 204-8588, Japan
}

\section{Supporting Information}

General. ${ }^{1} \mathrm{H}$ - and ${ }^{13} \mathrm{C}$-NMR spectra were recorded on a JEOL JNM-EX270, JNM-AL300, and JNM-GSX400 spectrometer. The chemical shifts were reported in ppm relative to $\mathrm{CHCl}_{3}(\delta=$ 7.26) for ${ }^{1} \mathrm{H}-\mathrm{NMR}$ and relative to the central $\mathrm{CDCl}_{3}$ resonance $(\delta=77.0)$ for ${ }^{13} \mathrm{C}$-NMR. UV-visible absorption spectra were measured with a Hitachi U-3210 spectrophotometer. IR spectra were recorded on a JASCO FT/IR-7000 spectrophotometer. Optical rotation was measured on a JASCO DIP-4 polarimeter. The mass spectroscopic data were obtained on a JEOL JNM-DX302 spectrometer. Cyclic voltammmetry was carried out at $25^{\circ} \mathrm{C}$ using BAS CV-50W electrochemical analyzer: working electrode, BAS-GCE glassy carbon disk $\left(7.07 \mathrm{~mm}^{2}\right.$ area); counter electrode, $\mathrm{Pt}$ wire $(0.1 \mathrm{~mm}$ diameter $)$; reference electrode, Ag wire (1 mm diameter). Chiral HPLC analysis was performed on a JASCO GULLIVER-series instrument.

Materials. Merck kiesel-gel 60 and Merck kiesel-gel $60 \mathrm{~F}_{254}$ were employed for silica gel column and thin layer chromatographies, respectively. 1,2-Dichloroethane and toluene were distilled from $\mathrm{P}_{2} \mathrm{O}_{5}$. THF and dioxane were distiled from benzophenone ketyl under a nitrogen atmosphere. Iron(III), manganese(III) and chromium(III) tetraphenylporphyrin complexes, $\mathrm{Fe}(\mathrm{TPP}) \mathrm{ClO}_{4},{ }^{1} \mathrm{Mn}(\mathrm{TPP}) \mathrm{ClO}_{4},{ }^{2} \mathrm{Cr}$ (TPP) $\mathrm{ClO}_{4},{ }^{3}$ and $\mathrm{Cr}(\mathrm{TPP}) \mathrm{Cl},{ }^{4}$ were prepared by the literature methods. Salen complexes, $(S, S)-(+)-N, N$ '-Bis(3,5-di-tert-butylsalicylidene)-1,2-cyclohexanediamino manganese(III) and chromium(III) triflates, Mn(salen)OTf and $\operatorname{Cr}($ salen)OTf, were prepared from the corresponding metallosalen chlorides, $\mathrm{Mn}$ (salen) $\mathrm{Cl}$ and $\mathrm{Cr}($ salen)Cl, respectively, by a counter ion exchange reaction using AgOTf in THF. Other chemicals were commercial products and were used without further purifications unless otherwise stated.

Preparation of tetraphenylporphynatochromium (III) Triflate, Cr(TPP)OTf. All glassware and a stirrer bar were dried overnight in an oven at $130^{\circ} \mathrm{C}$ and assembled while still warm. In two necked flask fitted with condenser was placed $\mathrm{Cr}(\mathrm{TPP}) \mathrm{Cl}(140 \mathrm{mg}, 0.2 \mathrm{mmol})$. The flask was sealed with septum, then was evacuated and backfilled with Ar. To the solid $\mathrm{Cr}(\mathrm{TPP}) \mathrm{Cl}$ was added anhydrous THF $(30 \mathrm{~mL})$, and the mixture was refluxed for $10 \mathrm{~min}$. To the refluxing solution was added AgOTf (62 mg, $0.24 \mathrm{mmol})$ in one portion, and the reaction mixture was continued to reflux under Ar until no $\mathrm{Cr}(\mathrm{TPP}) \mathrm{Cl}$ was found in the UV-visible spectrum of the solution (ca. $3 \mathrm{~h}$ ): absorption maxima shifted gradually from 603 and $450 \mathrm{~nm}$ to 598 and $452 \mathrm{~nm}$, respectively, during the axial ligand exchange reaction. The reaction mixture was allowed to cool to room temperature and then filtered through a Celite. The filtrate was concentrated under a reduced pressure, and the resulting residue was purified by recrystallization from $\mathrm{CH}_{2} \mathrm{Cl}_{2}-$ hexane (twice time) to give $\mathrm{Cr}(\mathrm{TPP}) \mathrm{OTf}$ as a dark green solid (77 mg, $46 \%$ ). UV-visible spectrum, cyclic voltammogram and IR spectrum are shown in Fig. 1, Fig. 2 and Fig. 3 on pages S8, S9, and S10, respectively. Anal. Calcd for $\mathrm{C}_{45} \mathrm{H}_{28} \mathrm{~N}_{4} \mathrm{~F}_{3} \mathrm{O}_{3} \mathrm{SCr} 1 / 2 \mathrm{H}_{2} \mathrm{O}: \mathrm{C}, 65.69 ; \mathrm{H}, 3.55 ; \mathrm{N}, 6.81$. Found: $\mathrm{C}$, $65.75 ; \mathrm{H}, 3.48 ; \mathrm{N}, 6.82$. 
Preparation of epoxides: Optical active epoxy silyl ethers $\mathbf{1 b}-\mathbf{4} \mathbf{f}^{5}$ and $\mathbf{1} \mathbf{g}^{6}$ were prepared by the reported methods. Other epoxides were prepared according to either of following procedures: (1) simple epoxidation of olefins with MCPBA; (2) VO(acac) $)_{2}$-catalyzed epoxidation of allylic alcohols with tert-BuOOH. ${ }^{5}$ The absolute configuration of epoxides $\mathbf{1 b} \mathbf{-} \mathbf{g}$ was determined by comparison of the optical rotations with those of authentic samples. ${ }^{5,6}$

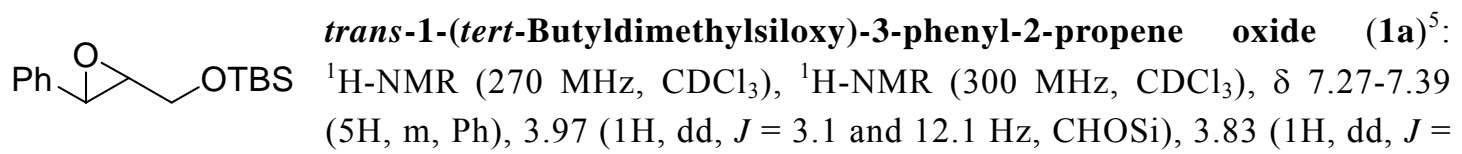
4.4 and $12.1 \mathrm{~Hz}, \mathrm{CHOSi}), 3.81(1 \mathrm{H}, \mathrm{d}, J=2.4 \mathrm{~Hz}, \mathrm{PhC} \underline{\mathrm{H}}), 3.13-3.17\left(1 \mathrm{H}, \mathrm{m}, \mathrm{CH}_{2} \mathrm{C} \underline{\mathrm{H}}-\mathrm{O}\right), 0.93(9 \mathrm{H}$, $\left.\mathrm{s},{ }^{t} \mathrm{Bu}\right), 0.11$ and 0.12 (each $3 \mathrm{H}, \mathrm{s},\left(\mathrm{CH}_{3}\right)_{2} \mathrm{Si}$ ).

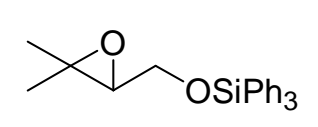

2,2-Dimethyl-3-(triphenylsiloxy)methyloxirane: ${ }^{1} \mathrm{H}-\mathrm{NMR}(270 \mathrm{MHz}$, $\left.\mathrm{CDCl}_{3}\right), \delta$ 7.35-7.66 $\left(15 \mathrm{H}, \mathrm{m}, \mathrm{Ph}_{3}\right), 3.94(1 \mathrm{H}, \mathrm{dd}, J=5.6$ and $11.2 \mathrm{~Hz}$, CHOSi), $3.84(1 \mathrm{H}, \mathrm{dd}, J=5.6$ and $11.2 \mathrm{~Hz}, \mathrm{CHOSi}), 3.00(1 \mathrm{H}, \mathrm{t}, J=5.6$ $\mathrm{Hz}, \mathrm{CH}-\mathrm{O}$ ), 1.29 and 1.34 (each $\left.3 \mathrm{H}, \mathrm{s},\left(\mathrm{CH}_{3}\right)_{2} \mathrm{C}\right) ;{ }^{13} \mathrm{C}-\mathrm{NMR}\left(100.4 \mathrm{MHz}, \mathrm{CDCl}_{3}\right), \delta 135.24$, 133.67, 130.02, 127.80, 63.59, 62.82, 24.76, 18.84; IR(KBr) $v / \mathrm{cm}^{-1}: 3068,3054,3044,2986$, 2952, 2936, 1591, 1487, 1458, 1429, 1381, 1267, 1247, 1139, 1118, 1073, 1054, 1029, 998, 866, 826, 779, 743, 714, 704, 679; Exact mass Calcd for $\mathrm{C}_{23} \mathrm{H}_{24} \mathrm{O}_{2} \mathrm{Si}$ : 360.1546, Found: 360.1543; Anal. Calcd for $\mathrm{C}_{23} \mathrm{H}_{24} \mathrm{O}_{2} \mathrm{Si}$ : C, 76.62; H, 6.71 Found: C, 76.52; H, 6.80.

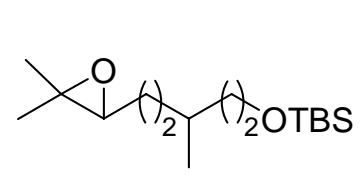

8-(tert-Butyldimethylsiloxy)-2,6-dimethyl-2-octene oxide ${ }^{5}:{ }^{1} \mathrm{H}-\mathrm{NMR}$ $\left(270 \mathrm{MHz}, \mathrm{CDCl}_{3}\right), \delta 3.56-3.63\left(2 \mathrm{H}, \mathrm{m}, \mathrm{CH}_{2} \mathrm{OSi}\right), 2.64(1 \mathrm{H}, \mathrm{t}, J=6.1$ $\mathrm{Hz}, \mathrm{CH}-\mathrm{O}), 1.22$ and 1.26 (each $\left.3 \mathrm{H}, \mathrm{s},\left(\mathrm{CH}_{3}\right)_{2} \mathrm{C}\right), 1.18-1.56(7 \mathrm{H}, \mathrm{m}, \mathrm{CH}$ and $\left.3 \mathrm{CH}_{2}\right), 0.85\left(3 \mathrm{H}, \mathrm{d}, J=8.9 \mathrm{~Hz}, \mathrm{CH}_{3}-\mathrm{CH}\right), 0.84\left(9 \mathrm{H}, \mathrm{s},{ }^{t} \mathrm{Bu}\right),-0.05$ and 0.00 (each $\left.3 \mathrm{H}, \mathrm{s},\left(\mathrm{CH}_{3}\right)_{2} \mathrm{Si}\right)$.

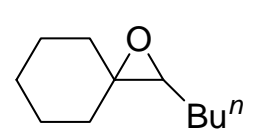

1-Cyclohexyliden-1-hexene oxide: ${ }^{1} \mathrm{H}-\mathrm{NMR}\left(270 \mathrm{MHz}, \mathrm{CDCl}_{3}\right), \delta 2.70$ $(1 \mathrm{H}, \mathrm{t}, J=5.9 \mathrm{~Hz}, \mathrm{CH}-\mathrm{O}), 1.20-1.80\left(16 \mathrm{H}, \mathrm{m}, 8 \mathrm{CH}_{2}\right), 0.92(3 \mathrm{H}, \mathrm{t}, J=6.9$ $\left.\mathrm{Hz}, \mathrm{CH}_{3}\right)$.<smiles>c1ccc(C23CCCCC2O3)cc1</smiles>

1-Phenylcyclohexene oxide: ${ }^{1} \mathrm{H}-\mathrm{NMR}\left(270 \mathrm{MHz}, \mathrm{CDCl}_{3}\right), \delta 7.21-7.41(5 \mathrm{H}, \mathrm{m}$, $\mathrm{Ph}), 3.06(1 \mathrm{H}, \mathrm{t}, J=2.2 \mathrm{~Hz}, \mathrm{CH}-\mathrm{O}), 1.96-2.34\left(4 \mathrm{H}, \mathrm{m}, 2 \mathrm{CH}_{2}\right), 1.23-1.67(4 \mathrm{H}, \mathrm{m}$, $\left.2 \mathrm{CH}_{2}\right)$.

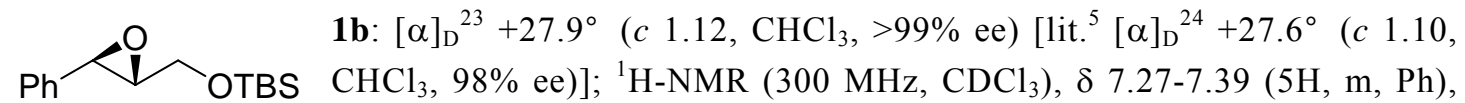
$3.97(1 \mathrm{H}, \mathrm{dd}, J=3.1$ and $12.1 \mathrm{~Hz}, \mathrm{CHOSi}), 3.83(1 \mathrm{H}, \mathrm{dd}, J=4.4$ and $12.1 \mathrm{~Hz}, \mathrm{CHOSi}), 3.81(1 \mathrm{H}$, $\mathrm{d}, J=2.4 \mathrm{~Hz}, \mathrm{PhC} \underline{\mathrm{H}}), 3.13-3.17\left(1 \mathrm{H}, \mathrm{m}, \mathrm{CH}_{2} \mathrm{C} \underline{\mathrm{H}}-\mathrm{O}\right), 0.93\left(9 \mathrm{H}, \mathrm{s},{ }^{t} \mathrm{Bu}\right), 0.11$ and 0.12 (each $3 \mathrm{H}, \mathrm{s}$, $\left.\left(\mathrm{CH}_{3}\right)_{2} \mathrm{Si}\right)$. The ee was determined by ${ }^{1} \mathrm{H}$ NMR analysis of the Mosher ester derived from the corresponding epoxy alcohol with (-)-MTPA chloride. 


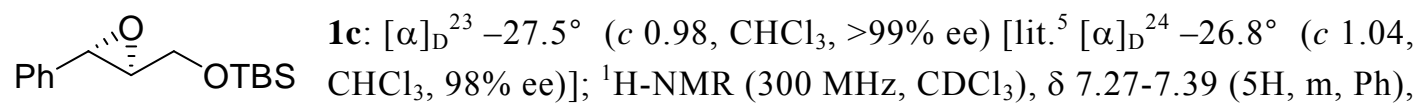
$3.97(1 \mathrm{H}, \mathrm{dd}, J=3.1$ and $12.1 \mathrm{~Hz}, \mathrm{CHOSi}), 3.83(1 \mathrm{H}, \mathrm{dd}, J=4.4$ and $12.1 \mathrm{~Hz}, \mathrm{CHOSi}), 3.81(1 \mathrm{H}$, $\mathrm{d}, J=2.4 \mathrm{~Hz}, \mathrm{PhC} \underline{\mathrm{H}}), 3.13-3.17\left(1 \mathrm{H}, \mathrm{m}, \mathrm{CH}_{2} \mathrm{C} \underline{\mathrm{H}}-\mathrm{O}\right), 0.93\left(9 \mathrm{H}, \mathrm{s},{ }^{t} \mathrm{Bu}\right), 0.11$ and 0.12 (each $3 \mathrm{H}, \mathrm{s}$, $\left.\left(\mathrm{CH}_{3}\right)_{2} \mathrm{Si}\right)$. The ee was determined by ${ }^{1} \mathrm{H}$ NMR analysis of the Mosher ester derived from the corresponding epoxy alcohol with (-)-MTPA chloride.

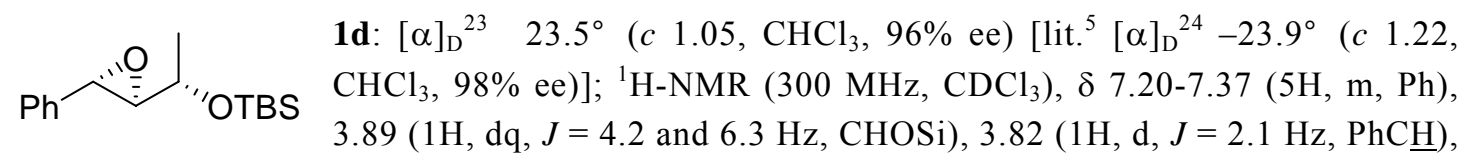
$2.92(1 \mathrm{H}, \mathrm{dd}, J=2.1$ and $4.2 \mathrm{~Hz}, \mathrm{CH}-\mathrm{O}), 1.29\left(3 \mathrm{H}, \mathrm{d}, J=6.3 \mathrm{~Hz}, \mathrm{CH}_{3}\right), 0.90\left(9 \mathrm{H}, \mathrm{s},{ }^{t} \mathrm{Bu}\right), 0.08$ and 0.09 (each $3 \mathrm{H}, \mathrm{s},\left(\mathrm{CH}_{3}\right)_{2} \mathrm{Si}$ ). The ee was determined by HPLC analysis (DAICEL CHIRACEL OD-H, 2-propanol/hexane $0.3 / 1000$, flow rate $0.5 \mathrm{~mL} / \mathrm{min}, t_{\mathrm{R}} 17.9 \mathrm{~min}$ (minor isomer) and 22.0 $\min$ (major isomer), detection at $220 \mathrm{~nm}$ ).

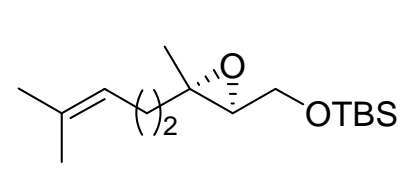

1e: $[\alpha]_{\mathrm{D}}{ }^{23}=-4.80^{\circ}\left(c 1.04, \mathrm{CHCl}_{3},>99 \%\right.$ ee $),\left[\right.$ lit. $^{5}[\alpha]_{\mathrm{D}}{ }^{24}=$ $-4.57^{\circ}$ (c $1.00, \mathrm{CHCl}_{3}, 95 \%$ ee)]; ${ }^{1} \mathrm{H}-\mathrm{NMR}\left(300 \mathrm{MHz}, \mathrm{CDCl}_{3}\right.$ ), $\delta$ $5.10(1 \mathrm{H}$, br t, $\mathrm{CH}=\mathrm{C}), 3.73\left(2 \mathrm{H}, \mathrm{dd}, J=1.2\right.$ and $\left.5.3 \mathrm{~Hz}, \mathrm{CH}_{2} \mathrm{OSi}\right)$, $2.90(1 \mathrm{H}, \mathrm{t}, J=5.3 \mathrm{~Hz}, \mathrm{CH}-\mathrm{O}), 2.08(2 \mathrm{H}, \mathrm{q}, J=7.2 \mathrm{~Hz}$, $\left.\mathrm{C}_{2}-\mathrm{CH}=\mathrm{C}\right), 1.61$ and 1.68 (each $\left.3 \mathrm{H}, \mathrm{s},\left(\mathrm{C}_{3}\right)_{2} \mathrm{C}=\mathrm{CH}\right), 1.40-1.65\left(2 \mathrm{H}, \mathrm{m}, \mathrm{CH}_{2} \mathrm{C}-\mathrm{O}\right), 1.27(3 \mathrm{H}, \mathrm{s}$, $\mathrm{CH}_{3} \mathrm{C}-\mathrm{O}$ ), $0.91\left(9 \mathrm{H}, \mathrm{s},{ }^{t} \mathrm{Bu}\right), 0.08$ and 0.09 (each $3 \mathrm{H}, \mathrm{s},\left(\mathrm{CH}_{3}\right)_{2} \mathrm{Si}$ ). The ee was determined by ${ }^{1} \mathrm{H}$ NMR analysis of the Mosher ester derived from the corresponding epoxy alcohol with (+)-MTPA chloride.

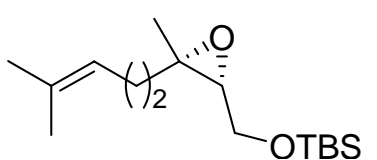

1f: $[\alpha]_{\mathrm{D}}^{23}=+4.18^{\circ}\left(c 0.97, \mathrm{CHCl}_{3}, 93 \%\right.$ ee $),\left[\operatorname{lit}^{5}{ }^{5}[\alpha]_{\mathrm{D}}{ }^{26}=+4.03^{\circ}(c\right.$ $1.07, \mathrm{CHCl}_{3}, 90 \%$ ee) $] ;{ }^{1} \mathrm{H}-\mathrm{NMR}\left(300 \mathrm{MHz}, \mathrm{CDCl}_{3}\right), \delta 5.10(1 \mathrm{H}$, br t, $\mathrm{CH}=\mathrm{C}), 3.77(1 \mathrm{H}, \mathrm{dd}, J=5.1$ and $11.7 \mathrm{~Hz}, \mathrm{CHOSi}), 3.69(1 \mathrm{H}, \mathrm{dd}, J=$

OTBS 5.7 and $11.7 \mathrm{~Hz}, \mathrm{CHOSi}), 2.88(1 \mathrm{H}, \mathrm{t}, J=5.1 \mathrm{~Hz}, \mathrm{CH}-\mathrm{O}), 2.11(2 \mathrm{H}, \mathrm{q}$, $\left.J=7.8 \mathrm{~Hz}, \underline{\mathrm{C}}_{2}-\mathrm{CH}=\mathrm{C}\right), 1.61$ and $1.69\left(\right.$ each $\left.3 \mathrm{H}, \mathrm{s},\left(\mathrm{C}_{3}\right)_{2} \mathrm{C}=\mathrm{CH}\right), 1.40-1.64\left(2 \mathrm{H}, \mathrm{m}, \mathrm{CH}_{2} \mathrm{C}-\mathrm{O}\right)$, $1.32\left(3 \mathrm{H}, \mathrm{s}, \mathrm{CH}_{3} \mathrm{C}-\mathrm{O}\right), 0.91\left(9 \mathrm{H}, \mathrm{s},{ }^{\mathrm{t}} \mathrm{Bu}\right), 0.08$ and 0.09 (each $\left.3 \mathrm{H}, \mathrm{s},\left(\mathrm{CH}_{3}\right)_{2} \mathrm{Si}\right)$. The ee was determined by ${ }^{1} \mathrm{H}$ NMR analysis of the Mosher ester derived from the corresponding epoxy alcohol with (+)-MTPA chloride.

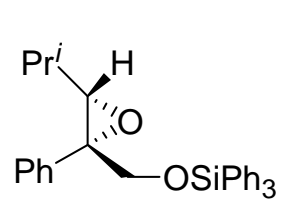

1g: $[\alpha]_{\mathrm{D}}^{23}=-24.8^{\circ}\left(c 1.04, \mathrm{CHCl}_{3}\right),\left[\right.$ lit. $^{6}[\alpha]_{\mathrm{D}}{ }^{26}=-24.8^{\circ}\left(\mathrm{c} 1.83, \mathrm{CHCl}_{3}\right.$, $>99 \%$ ee)]; ${ }^{1} \mathrm{H}-\mathrm{NMR}\left(300 \mathrm{MHz}, \mathrm{CDCl}_{3}\right): \delta 7.27-7.59(20 \mathrm{H}, \mathrm{m}, \mathrm{Ph}), 4.08(2 \mathrm{H}$, $\left.\mathrm{s}, \mathrm{CH}_{2} \mathrm{OSi}\right), 2.94(1 \mathrm{H}, \mathrm{d}, J=8.6 \mathrm{~Hz}, \mathrm{CH}-\mathrm{O}), 0.92-0.99\left(4 \mathrm{H}, \mathrm{m}, \mathrm{CH}_{3}\right.$ and $\left.\mathrm{C} \underline{\mathrm{H}}\left(\mathrm{CH}_{3}\right)_{2}\right), 0.74\left(3 \mathrm{H}, \mathrm{d}, J=6.6 \mathrm{~Hz}, \mathrm{CH}_{3}\right)$. The ee was determined by ${ }^{1} \mathrm{H}$ NMR analysis of the Mosher ester derived from the corresponding epoxy alcohol with (-)-MTPA chloride. 
Typical procedure for the $\mathrm{Cr}$ (TPP)OTf-catalyzed rearrangements of epoxides to aldehydes. The catalyst $\mathrm{Cr}$ (TPP)OTf were dried over silica gel for $10 \mathrm{~h}$ under reduced pressure $(1 \mathrm{mmHg})$ at $100^{\circ} \mathrm{C}$ just before its use. To a solution of epoxides 1 (1 mmol) in freshly distilled 1,2-dichloroethane $(5 \mathrm{~mL})$ was added $\operatorname{Cr}(\mathrm{TPP}) \mathrm{OTf}(0.01 \mathrm{mmol})$. The mixture was heated to reflux under Ar. After completion of the reaction (monitored by tlc analysis), the reaction mixture was concentrated under a reduced pressure. The residue was passed through a silica gel short column (1:3 AcOEt- hexane) to remove the catalyst. The eluate was concentrated in vacuo, and the residue was chromatographed on silica-gel (1:3 AcOEt-hexane) to give aldehydes 3 . The absolute configuration of the products was determined by comparison of the optical rotations with those of authentic samples. ${ }^{5,6}$

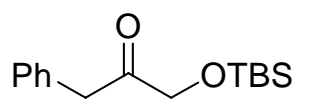

2a: ${ }^{1} \mathrm{H}-\mathrm{NMR}\left(270 \mathrm{MHz}, \mathrm{CDCl}_{3}\right) \delta 7.13-7.29(5 \mathrm{H}, \mathrm{m}, \mathrm{Ph}), 4.17(2 \mathrm{H}, \mathrm{s}$, $\left.\mathrm{CH}_{2} \mathrm{OSi}\right), 3.75\left(2 \mathrm{H}, \mathrm{s}, \mathrm{CH}_{2} \mathrm{Ph}\right), 0.86(9 \mathrm{H}, \mathrm{s}$, tert- $\mathrm{Bu}), 0.00(6 \mathrm{H}, \mathrm{s}$, $\left.\left(\mathrm{CH}_{3}\right)_{2} \mathrm{Si}\right)$.<smiles>O=CC(C[SeH2])c1ccccc1</smiles>

3a ${ }^{5}:{ }^{1} \mathrm{H}-\mathrm{NMR}\left(270 \mathrm{MHz}, \mathrm{CDCl}_{3}\right), \delta 9.82(1 \mathrm{H}, \mathrm{d}, J=2.0 \mathrm{~Hz}, \mathrm{CHO}), 7.20-7.65$ $(5 \mathrm{H}, \mathrm{m}, \mathrm{Ph}), 4.24(1 \mathrm{H}, \mathrm{dd}, J=7.4$ and $10.1 \mathrm{~Hz}, \mathrm{CHOSi}), 3.96(1 \mathrm{H}, \mathrm{dd}, J=5.6$ and $10.1 \mathrm{~Hz}, \mathrm{CHOSi}), 3.74(1 \mathrm{H}$, br t, $J=6.5 \mathrm{~Hz}, \mathrm{PhCH}), 0.85(9 \mathrm{H}, \mathrm{s}$, tert-Bu), -0.02 and 0.00 (each $3 \mathrm{H}, \mathrm{s},\left(\mathrm{CH}_{3}\right)_{2} \mathrm{Si}$ ).

2,2-Dimethyl-3-triphenylsiloxypropanal: ${ }^{1} \mathrm{H}-\mathrm{NMR}\left(300 \mathrm{MHz}, \mathrm{CDCl}_{3}\right), \delta 9.57$<smiles>CC(C)(C=O)CO[SnH3]</smiles>
$(1 \mathrm{H}, \mathrm{s}, \mathrm{CHO}), 7.26-7.65\left(15 \mathrm{H}, \mathrm{m}, \mathrm{Ph}_{3}\right), 3.78\left(2 \mathrm{H}, \mathrm{s}, \mathrm{CH}_{2} \mathrm{OSi}\right), 1.07(6 \mathrm{H}, \mathrm{s}$, $\left.\left(\mathrm{CH}_{3}\right)_{2} \mathrm{C}\right) ;{ }^{13} \mathrm{C}-\mathrm{NMR}\left(100.4 \mathrm{MHz}, \mathrm{CDCl}_{3}\right), \delta 205.23,135.22,133.47,130.04$, 127.80, 68.72, 48.36, 18.78; IR(KBr) $\vee / \mathrm{cm}^{-1}: 3076,3054,3002,2962,2926$, 2854, 1734, 1591, 1487, 1431, 1265, 1118, 1031, 893, 824, 745, 735, 714, 702; Exact mass Calcd for $\mathrm{C}_{23} \mathrm{H}_{24} \mathrm{O}_{2} \mathrm{Si}: 360.1546$. Found: 360.1537 .

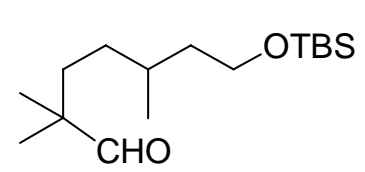

7-(tert-Butyldimethylsiloxy)-2,2,5-trimethylheptanal ${ }^{5}: \quad{ }^{1} \mathrm{H}-\mathrm{NMR}$ $\left(270 \mathrm{MHz}, \mathrm{CDCl}_{3}\right), \delta 9.43(1 \mathrm{H}, \mathrm{s}, \mathrm{CHO}), 3.54-3.68\left(2 \mathrm{H}, \mathrm{m}, \mathrm{CH}_{2} \mathrm{OSi}\right)$, 1.10-1.59 (7H, m, $\mathrm{CHCH}_{3}$ and $\left.3 \mathrm{CH}_{2}\right), 1.03\left(6 \mathrm{H}, \mathrm{s},\left(\mathrm{CH}_{3}\right){ }_{2} \mathrm{C}\right), 0.88$ $(9 \mathrm{H}, \mathrm{s}$, tert- $\mathrm{Bu}), 0.87\left(3 \mathrm{H}, \mathrm{d}, J=6.6 \mathrm{~Hz}, \mathrm{C}_{3} \mathrm{CH}\right), 0.03(6 \mathrm{H}, \mathrm{s}$, $\left.\left(\mathrm{CH}_{3}\right)_{2} \mathrm{Si}\right)$; Anal. Calcd for $\mathrm{C}_{16} \mathrm{H}_{34} \mathrm{O}_{2} \mathrm{Si}$ : C, 67.07; H, 11.96. Found: C, 66.98; H, 11.98.

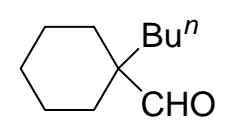

1-Butylcyclohexanecarbaldehyde: ${ }^{1} \mathrm{H}-\mathrm{NMR}\left(270 \mathrm{MHz}, \mathrm{CDCl}_{3}\right), \delta 9.42(1 \mathrm{H}$, $\mathrm{s}, \mathrm{CHO}), 1.80-1.95\left(2 \mathrm{H}, \mathrm{m}, \mathrm{CH}_{2}\right), 1.05-1.68\left(14 \mathrm{H}, \mathrm{m}, 7 \mathrm{CH}_{2}\right), 0.87(3 \mathrm{H}, \mathrm{t}, J=$ $\left.6.9 \mathrm{~Hz}, \mathrm{CH}_{3}\right) ;{ }^{13} \mathrm{C}-\mathrm{NMR}\left(67.8 \mathrm{MHz}, \mathrm{CDCl}_{3}\right), \delta 207.42,49.70,36.32,31.07$, $25.88,25.64,23.36,22.64,13.91$.

1-Phenylcyclopentanecarbaldehyde: ${ }^{1} \mathrm{H}-\mathrm{NMR}\left(270 \mathrm{MHz}, \mathrm{CDCl}_{3}\right), \delta 9.40(1 \mathrm{H}$, s, CHO), 7.23-7.39 (5H, m, Ph), 2.48-2.57 (2H, m, $\left.\mathrm{CH}_{2}\right), 1.57-1.94(6 \mathrm{H}, \mathrm{m})$. 


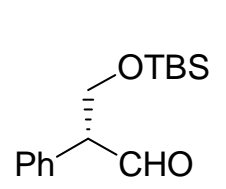

3b: $[\alpha]_{\mathrm{D}}^{23}+32.6^{\circ}\left(c 1.03, \mathrm{CHCl}_{3},>99 \%\right.$ ee $)\left[\right.$ lit. $^{5}[\alpha]_{\mathrm{D}}^{22}+32.3^{\circ}\left(c 1.00, \mathrm{CHCl}_{3}\right.$, $98 \%$ ee)]; ${ }^{1} \mathrm{H}-\mathrm{NMR}\left(300 \mathrm{MHz}, \mathrm{CDCl}_{3}\right), \delta 9.84(1 \mathrm{H}, \mathrm{d}, J=2.0 \mathrm{~Hz}, \mathrm{CHO})$, 7.22-7.41 $(5 \mathrm{H}, \mathrm{m}, \mathrm{Ph}), 4.25(1 \mathrm{H}, \mathrm{dd}, J=7.3$ and $10.1 \mathrm{~Hz}, \mathrm{CHOSi}), 3.98(1 \mathrm{H}, \mathrm{dd}$, $J=5.7$ and $10.1 \mathrm{~Hz}, \mathrm{CHOSi}), 3.75(1 \mathrm{H}, \mathrm{br} \mathrm{t}, J=6.6 \mathrm{~Hz}, \mathrm{PhC} \underline{\mathrm{H}}), 0.85(9 \mathrm{H}, \mathrm{s}$, ${ }^{t} \mathrm{Bu}$ ), -0.01 and 0.01 (each $3 \mathrm{H}, \mathrm{s},\left(\mathrm{CH}_{3}\right)_{2} \mathrm{Si}$ ). The ee was determined by HPLC analysis after the conversion of aldehyde $\mathbf{3 b}$ to the corresponding alcohol with $\mathrm{NaBH}_{4}$ and then to the benzoic ester with PhCOCl, DMAP, and $\mathrm{Et}_{3} \mathrm{~N}$ (DAICEL CHIRALPAK AD-H, 2-propanol/hexane 1/500, flow rate $0.5 \mathrm{~mL} / \mathrm{min}, t_{\mathrm{R}} 18.3 \mathrm{~min}$ (minor isomer) and $19.4 \mathrm{~min}$ (major isomer), detection at $220 \mathrm{~nm}$ ).

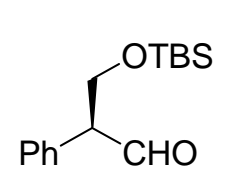

3c: $[\alpha]_{\mathrm{D}}^{23}-32.5^{\circ}\left(c 1.15, \mathrm{CHCl}_{3},>99 \%\right.$ ee $)\left[\right.$ lit. $^{5}[\alpha]_{\mathrm{D}}{ }^{21}-31.8^{\circ}\left(c 1.00, \mathrm{CHCl}_{3}\right.$, $98 \%$ ee)]; ${ }^{1} \mathrm{H}-\mathrm{NMR}\left(300 \mathrm{MHz}, \mathrm{CDCl}_{3}\right), \delta 9.84(1 \mathrm{H}, \mathrm{d}, J=2.0 \mathrm{~Hz}, \mathrm{CHO})$, 7.22-7.41 $(5 \mathrm{H}, \mathrm{m}, \mathrm{Ph}), 4.25(1 \mathrm{H}, \mathrm{dd}, J=7.3$ and $10.1 \mathrm{~Hz}, \mathrm{CHOSi}), 3.97(1 \mathrm{H}$, dd, $J=5.7$ and $10.1 \mathrm{~Hz}, \mathrm{CHOSi}), 3.75(1 \mathrm{H}$, br t, $J=6.5 \mathrm{~Hz}, \mathrm{PhCH}), 0.85(9 \mathrm{H}, \mathrm{s}$,

${ }^{t} \mathrm{Bu}$ ), -0.01 and 0.01 (each $3 \mathrm{H}, \mathrm{s},\left(\mathrm{CH}_{3}\right)_{2} \mathrm{Si}$ ). The ee was determined by HPLC analysis after the conversion of aldehyde $\mathbf{3 c}$ to the corresponding alcohol with $\mathrm{NaBH}_{4}$ and then to the benzoic ester with PhCOCl, DMAP, and $\mathrm{Et}_{3} \mathrm{~N}$ (DAICEL CHIRALPAK AD-H, 2-propanol/hexane 1/500, flow rate $0.5 \mathrm{~mL} / \mathrm{min}, t_{\mathrm{R}} 18.1 \mathrm{~min}$ (major isomer) and $19.7 \mathrm{~min}$ (minor isomer), detection at $220 \mathrm{~nm}$ ).

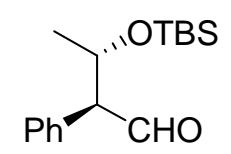

3d: $[\alpha]_{\mathrm{D}}^{23}=+64.0^{\circ}\left(c 1.07, \mathrm{CHCl}_{3}, 96 \%\right.$ ee $),\left[\right.$ lit. $^{5}[\alpha]_{\mathrm{D}}{ }^{22}=+64.8^{\circ}(c$ 1.12, $\left.\mathrm{CHCl}_{3}\right), 98 \%$ ee]; ${ }^{1} \mathrm{H}-\mathrm{NMR}\left(300 \mathrm{MHz}, \mathrm{CDCl}_{3}\right), \delta 9.88(1 \mathrm{H}, \mathrm{d}, J=3.3 \mathrm{~Hz}, \mathrm{CHO})$, 7.20-7.39 $(5 \mathrm{H}, \mathrm{m}, \mathrm{Ph}), 4.50(1 \mathrm{H}, \mathrm{dq}, J=6.1$ and $8.4 \mathrm{~Hz}, \mathrm{CHOSi}), 3.53(1 \mathrm{H}, \mathrm{dd}$, $J=3.3$ and $8.4 \mathrm{~Hz}, \mathrm{C} \underline{\mathrm{HCHO}}), 1.07\left(3 \mathrm{H}, \mathrm{d}, J=6.1 \mathrm{~Hz}, \mathrm{CH}_{3}\right), 0.89\left(9 \mathrm{H}, \mathrm{s},{ }^{t} \mathrm{Bu}\right)$, 0.08 and 0.09 (each $3 \mathrm{H}, \mathrm{s},\left(\mathrm{CH}_{3}\right)_{2} \mathrm{Si}$ ). The ee was determined by HPLC analysis after the conversion of aldehyde $\mathbf{3 d}$ to the corresponding alcohol with $\mathrm{NaBH}_{4}$ and then to the benzoic ester with PhCOCl, DMAP, and $\mathrm{Et}_{3} \mathrm{~N}$ (DAICEL CHIRACEL OD-H, 2-propanol/hexane 0.2/500, flow rate $0.5 \mathrm{~mL} / \mathrm{min}, t_{\mathrm{R}} 29.4 \mathrm{~min}$ (major isomer) and $31.0 \mathrm{~min}$ (minor isomer), detection at $220 \mathrm{~nm}$ ).

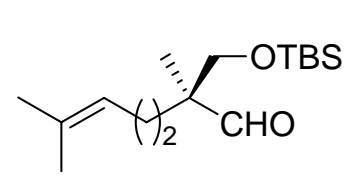

3e from silyl ether of epoxy geraniol: $[\alpha]_{\mathrm{D}}^{23}=+6.78^{\circ}\left(c 1.06, \mathrm{CHCl}_{3}\right.$, $>99 \%$ ee $),\left[\right.$ lit. $^{5}[\alpha]_{\mathrm{D}}{ }^{24}=+6.45^{\circ}\left(c \quad 1.00, \mathrm{CHCl}_{3}\right), 95 \%$ ee $] ;{ }^{1} \mathrm{H}-\mathrm{NMR}$ $\left(300 \mathrm{MHz}, \mathrm{CDCl}_{3}\right), \delta 9.56(1 \mathrm{H}, \mathrm{s}, \mathrm{CHO}), 5.06(1 \mathrm{H}$, br t, $\mathrm{CH}=\mathrm{C}), 3.68$ $(1 \mathrm{H}, \mathrm{d}, J=9.9 \mathrm{~Hz}, \mathrm{CHOSi}), 3.57(1 \mathrm{H}, \mathrm{d}, J=9.9 \mathrm{~Hz}, \mathrm{CHOSi}), 1.85-1.96$ $\left(2 \mathrm{H}, \mathrm{m}, \mathrm{C}_{2}-\mathrm{CH}=\mathrm{C}\right), 1.58$ and 1.67 (each $\left.3 \mathrm{H}, \mathrm{s},\left(\underline{\mathrm{C}}_{3}\right)_{2} \mathrm{C}=\mathrm{CH}\right), 1.41-1.63\left(2 \mathrm{H}, \mathrm{m}, \mathrm{C}_{2}-\mathrm{C}-\mathrm{CHO}\right)$, $1.04\left(3 \mathrm{H}, \mathrm{s}, \mathrm{CH}_{3}\right), 0.87\left(9 \mathrm{H}, \mathrm{s},{ }^{t} \mathrm{Bu}\right), 0.03\left(6 \mathrm{H}, \mathrm{s},\left(\mathrm{CH}_{3}\right)_{2} \mathrm{Si}\right)$. The ee was determined by HPLC analysis after the conversion of aldehyde $3 \mathbf{e}$ to the corresponding alcohol with $\mathrm{NaBH}_{4}$ and then to the $p$-nitrobenzoic ester with $p-\mathrm{NO}_{2}-\mathrm{PhCOCl}$, DMAP, and $\mathrm{Et}_{3} \mathrm{~N}$ (DAICEL CHIRACEL OD-H, 2-propanol/hexane 1/500, flow rate $0.4 \mathrm{~mL} / \mathrm{min}, t_{\mathrm{R}} 19.4 \mathrm{~min}$ (major isomer) and $20.2 \mathrm{~min}$ (minor isomer), detection at $250 \mathrm{~nm}$ ).

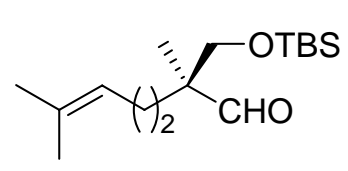

3e from silyl ether of epoxy nerol: $[\alpha]_{\mathrm{D}}^{23}=+6.35^{\circ}$ (c 1.04, $\mathrm{CHCl}_{3}$, $93 \%$ ee $),\left[\right.$ lit. $^{5}[\alpha]_{\mathrm{D}}{ }^{24}=+6.45^{\circ}\left(c 1.00, \mathrm{CHCl}_{3}\right), 95 \%$ ee $] ;{ }^{1} \mathrm{H}-\mathrm{NMR}(300$ $\left.\mathrm{MHz}, \mathrm{CDCl}_{3}\right), \delta 9.56(1 \mathrm{H}, \mathrm{s}, \mathrm{CHO}), 5.06(1 \mathrm{H}, \mathrm{br} \mathrm{t}, \mathrm{CH}=\mathrm{C}), 3.68(1 \mathrm{H}, \mathrm{d}$, $J=9.9 \mathrm{~Hz}, \mathrm{CHOSi}), 3.57(1 \mathrm{H}, \mathrm{d}, J=9.9 \mathrm{~Hz}, \mathrm{CHOSi}), 1.85-1.95(2 \mathrm{H}, \mathrm{m}$,

$\left.\mathrm{C}_{2}-\mathrm{CH}=\mathrm{C}\right), 1.58$ and 1.68 (each $\left.3 \mathrm{H}, \mathrm{s},\left(\mathrm{C}_{3}\right)_{2} \mathrm{C}=\mathrm{CH}\right), 1.41-1.64\left(2 \mathrm{H}, \mathrm{m}, \mathrm{C}_{2}-\mathrm{C}-\mathrm{CHO}\right), 1.04(3 \mathrm{H}$, $\left.\mathrm{s}, \mathrm{CH}_{3}\right), 0.87\left(9 \mathrm{H}, \mathrm{s},{ }^{t} \mathrm{Bu}\right), 0.03\left(6 \mathrm{H}, \mathrm{s},\left(\mathrm{CH}_{3}\right)_{2} \mathrm{Si}\right)$. The ee was determined by HPLC analysis after the conversion of aldehyde $3 \mathbf{e}$ to the corresponding alcohol with $\mathrm{NaBH}_{4}$ and then to the 
$p$-nitrobenzoic ester with $p-\mathrm{NO}_{2}-\mathrm{PhCOCl}$, DMAP, and $\mathrm{Et}_{3} \mathrm{~N}$ (DAICEL CHIRACEL OD-H, 2-propanol/hexane 1/500, flow rate $0.4 \mathrm{~mL} / \mathrm{min}, t_{\mathrm{R}} 19.1 \mathrm{~min}$ (major isomer) and $19.9 \mathrm{~min}$ (minor isomer), detection at $220 \mathrm{~nm}$ ).

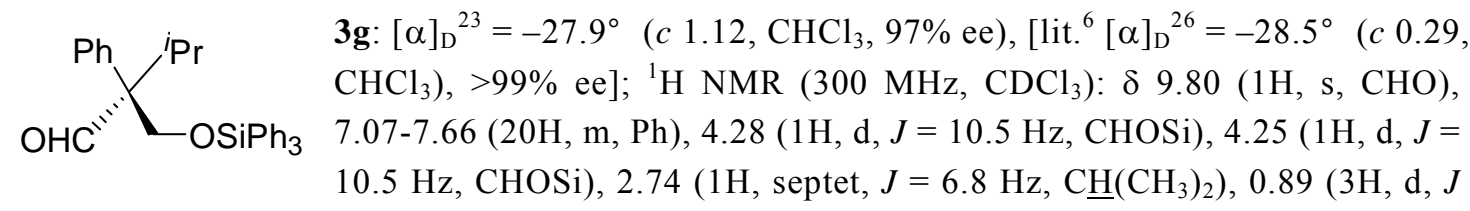
$\left.=6.8 \mathrm{~Hz}, \mathrm{CH}_{3}\right), 0.81\left(3 \mathrm{H}, \mathrm{d}, J=6.8 \mathrm{~Hz}, \mathrm{CH}_{3}\right)$. The ee was determined by HPLC analysis after the conversion of aldehyde $\mathbf{3 g}$ to the corresponding alcohol with $\mathrm{NaBH}_{4}$ (DAICEL CHIRACEL OD-H, 2-propanol/hexane $0.2 / 500$, flow rate $0.4 \mathrm{~mL} / \mathrm{min}, t_{\mathrm{R}} 14.2 \mathrm{~min}$ (minor isomer) and $15.0 \mathrm{~min}$ (major isomer), detection at $220 \mathrm{~nm}$ ).

\section{References}

(1) Reed, C. A.; Mashiko, T.; Bentley, S. P.; Kastner, M. E.; Scheidt, W. R.; Spartalian, K.; Lang, G. J. Am. Chem. Soc., 1979, 101, 2948.

(2) Kelly, S. L.; Kadish, K. M. Inorg. Chem. 1982, 21, 3631.

(3) Kelly, S. L.; Kadish, K. M. Inorg., Chem., 1984, 23, 679.

(4) Preparation and characterization of $\mathrm{Cr}(\operatorname{tpp}) \mathrm{Cl}$, see: (a) Summerville, D. A.; Jones, R. D.; Hoffman, B. M.; Basolo, F. J. Am. Chem. Soc., 1977, 99, 8195. (b) Bottomley, L. A.; Kadish, K. M. J. Chem. Soc., Chem. Commun., 1981, 1212.

(5) Maruoka, K.; Ooi, T.; Nagahara, S.; Yamamoto, H. Tetrahedron, 1991, 47, 6983.

(6) Kimura, T.; Yamamoto, N.; Suzuki, Y.; Kawano, K.; Norimine, Y.; Ito, K.; Nagato, S.; Iimura, Y.; Yonaga, M. J. Org. Chem., 2002, 67, 6228. 


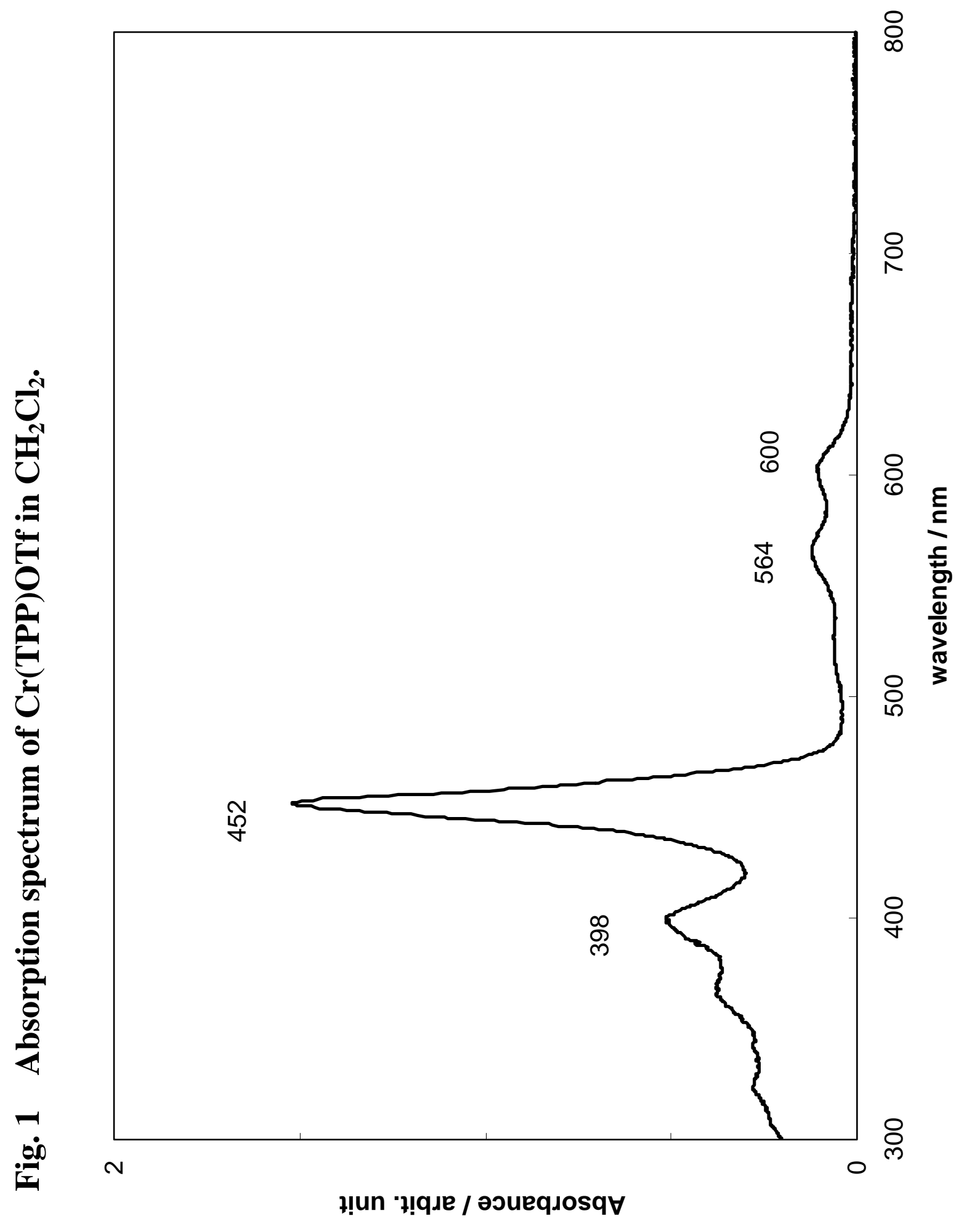



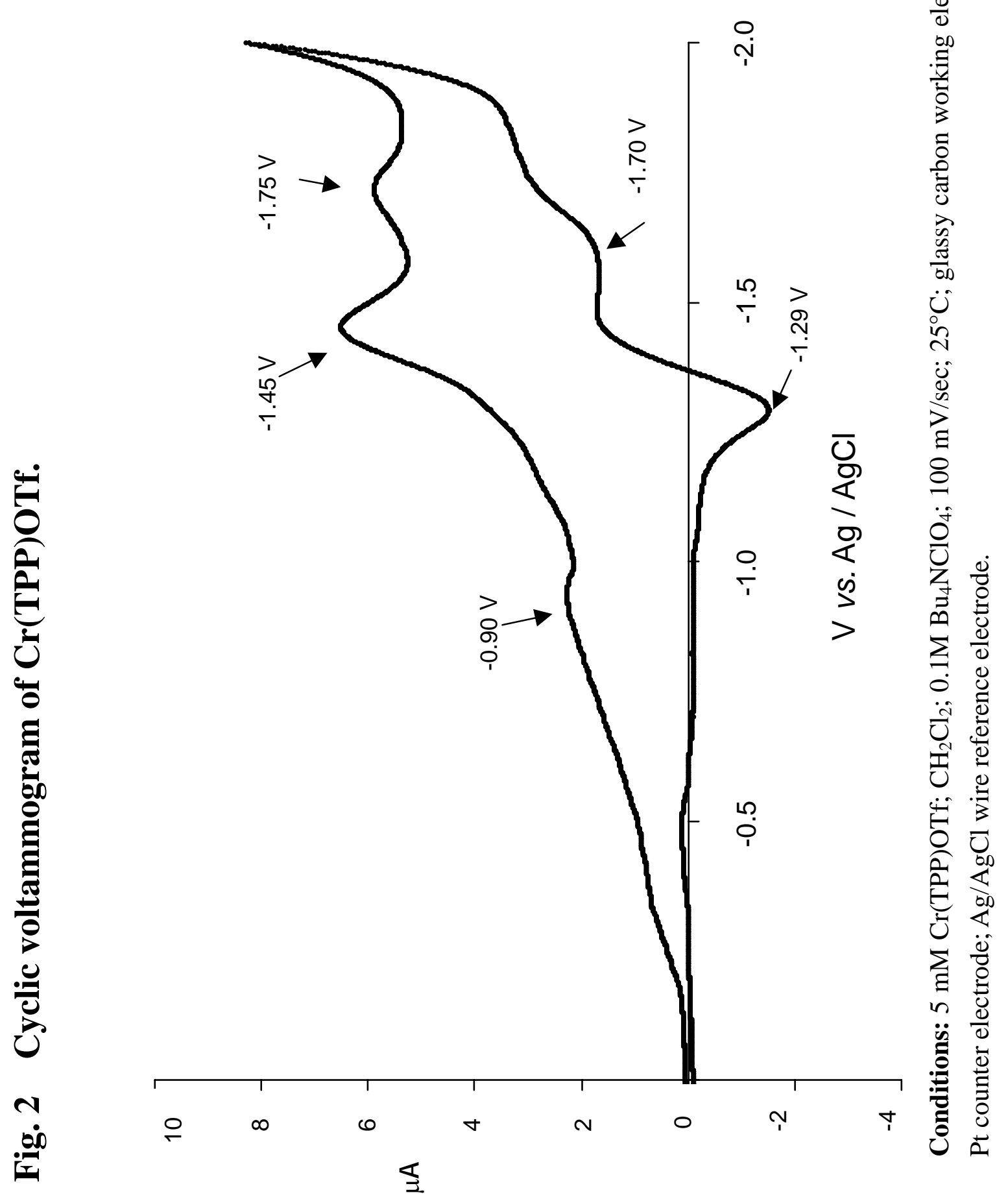


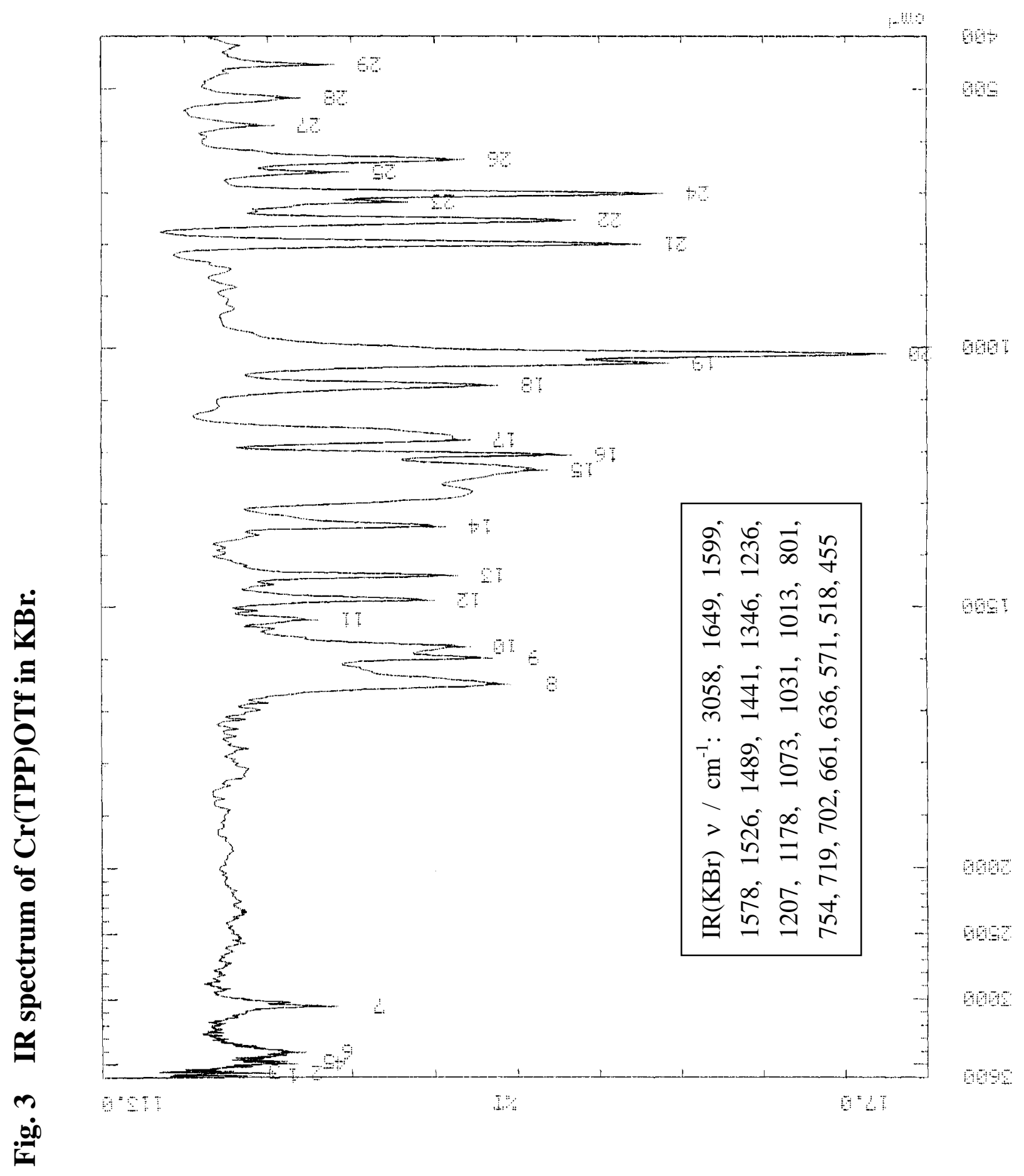




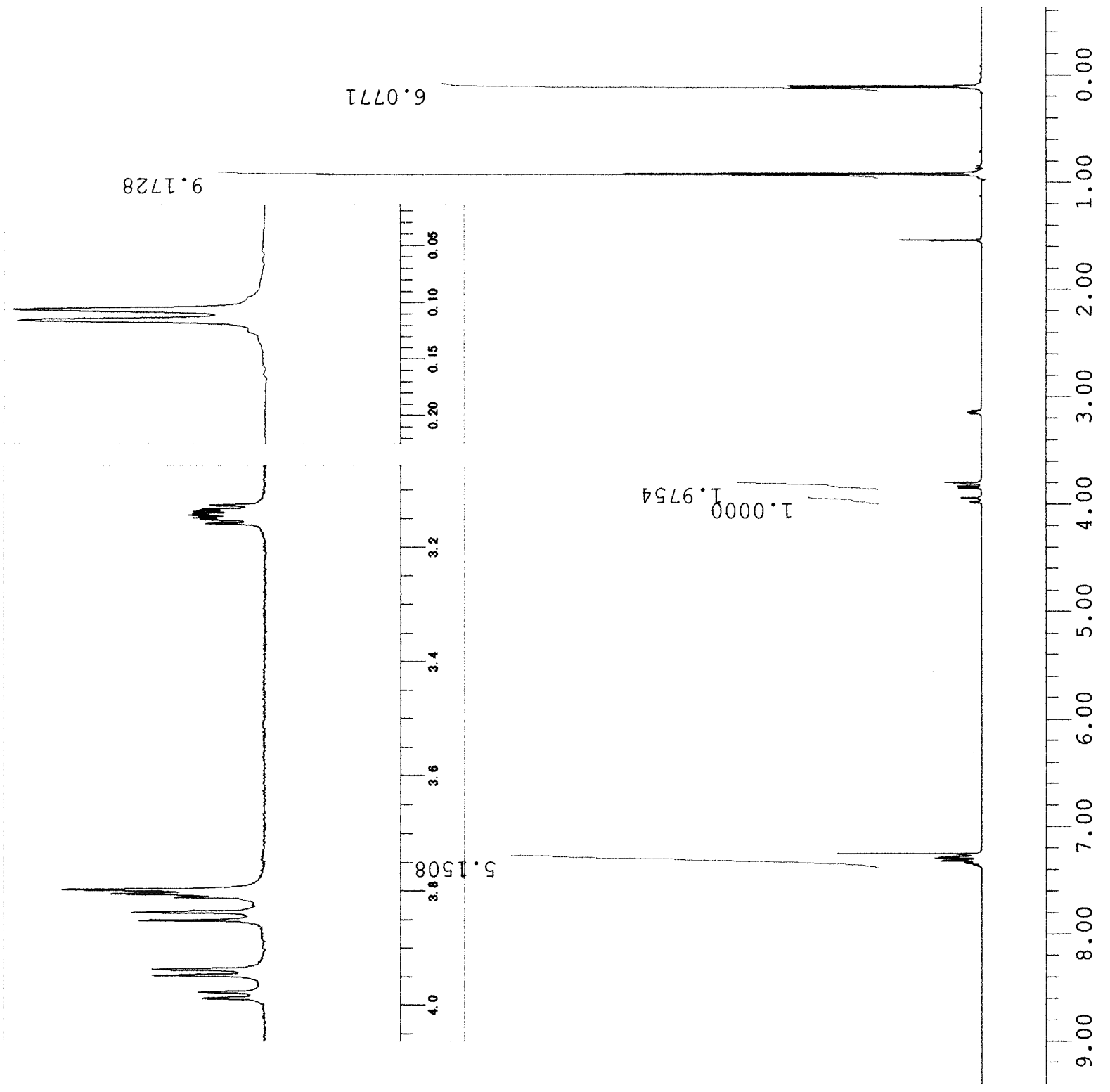

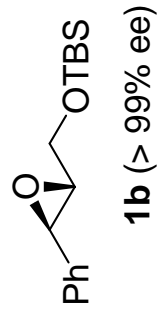




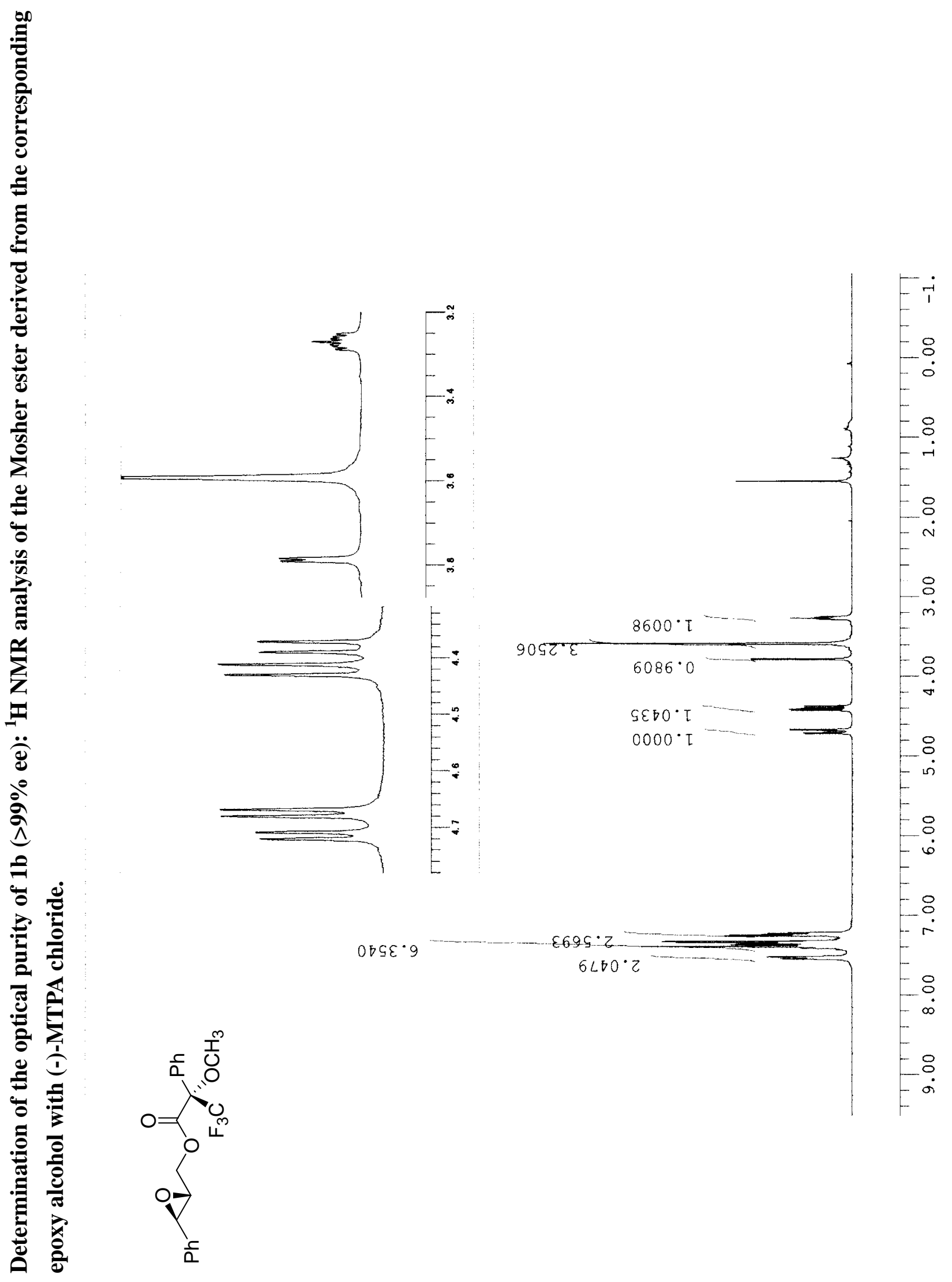




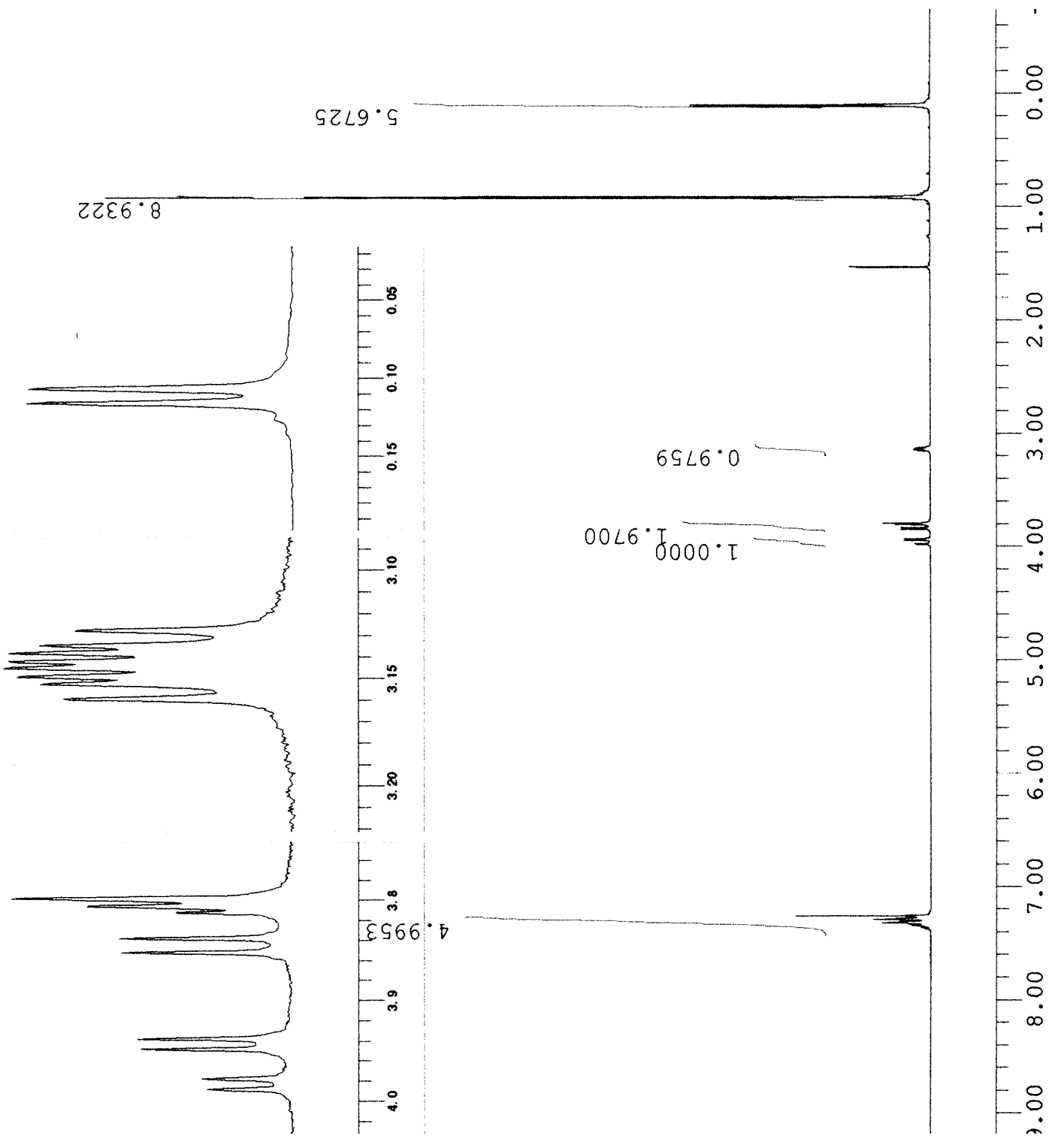

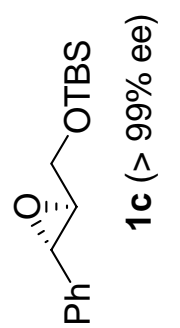




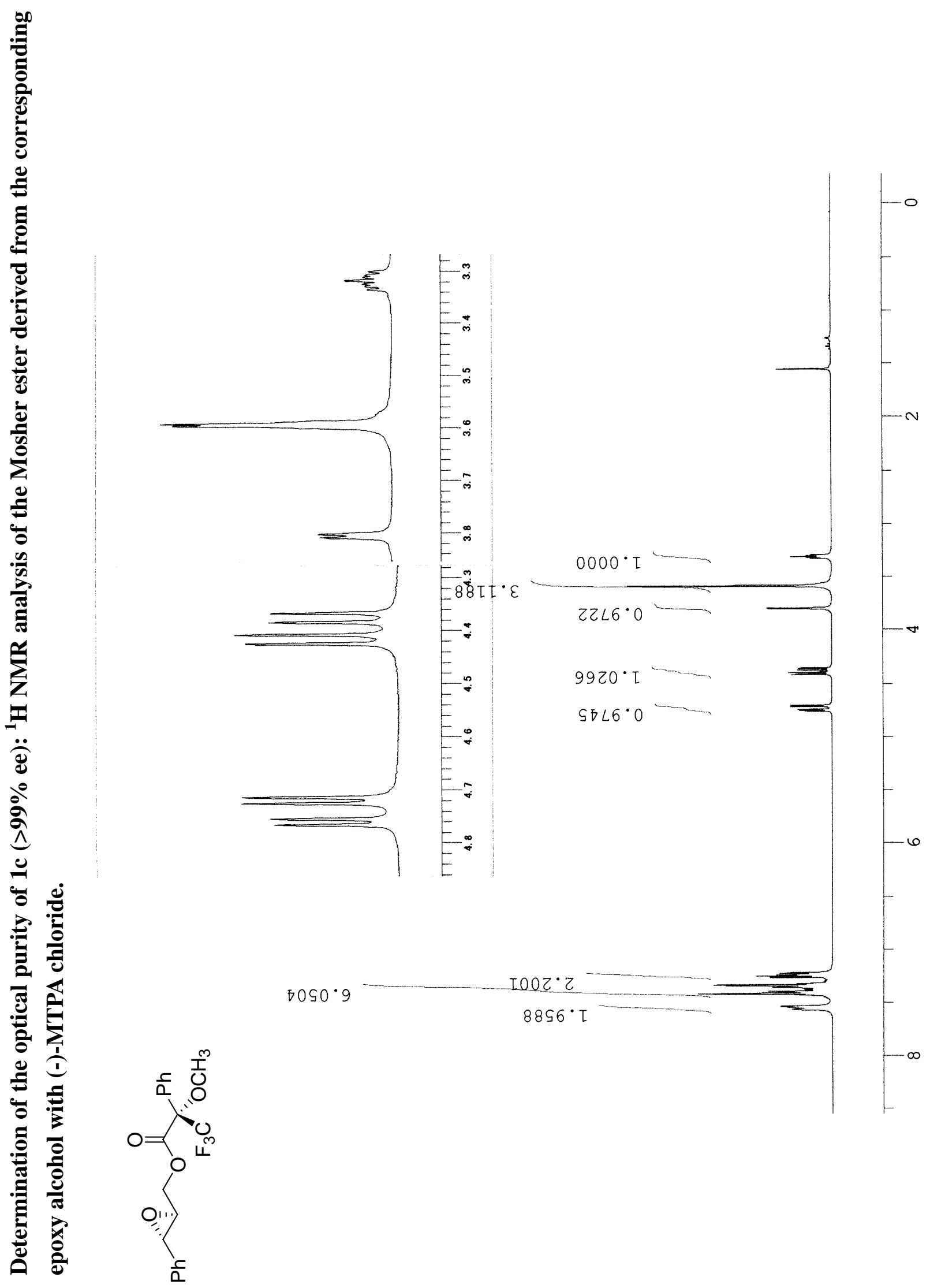




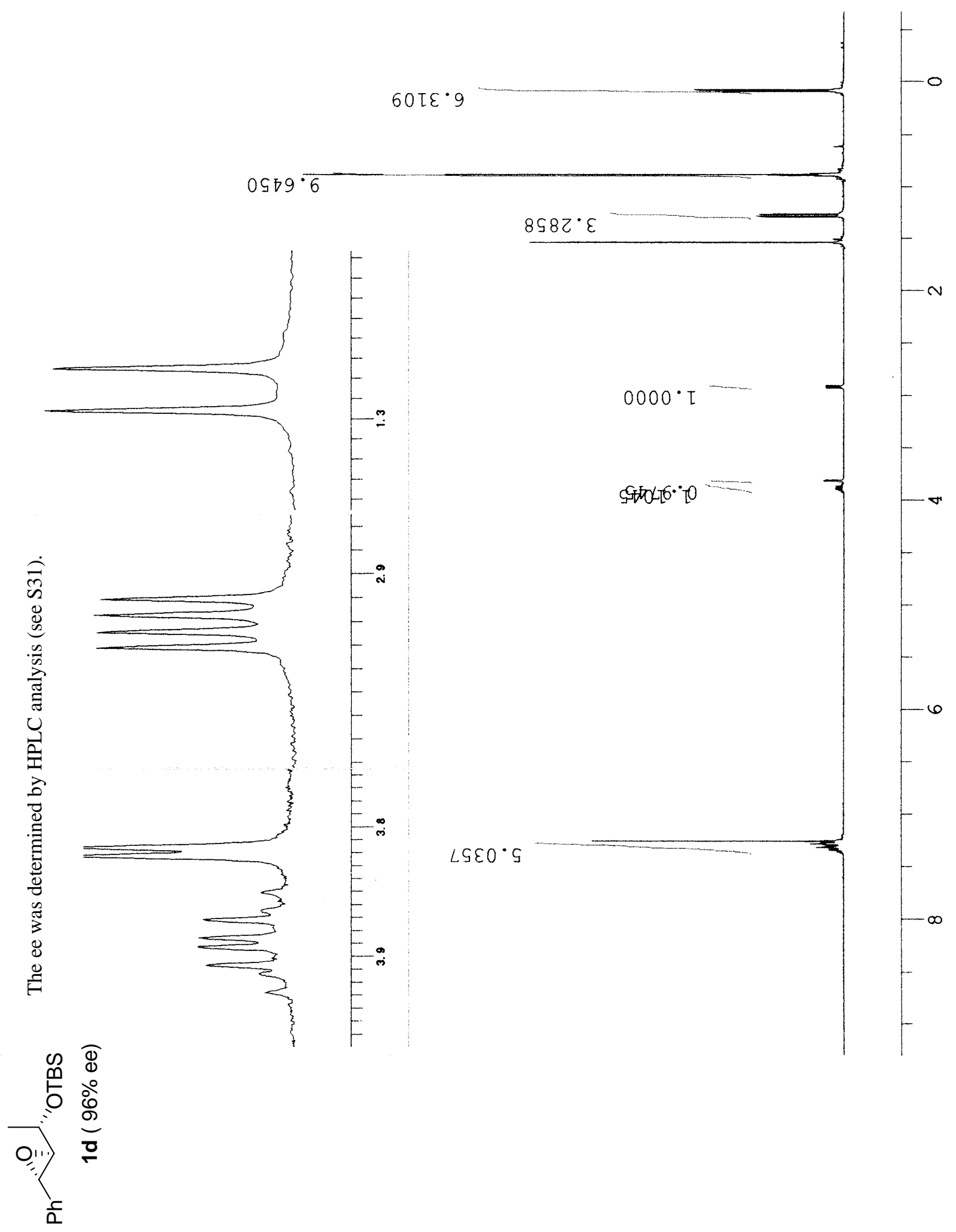




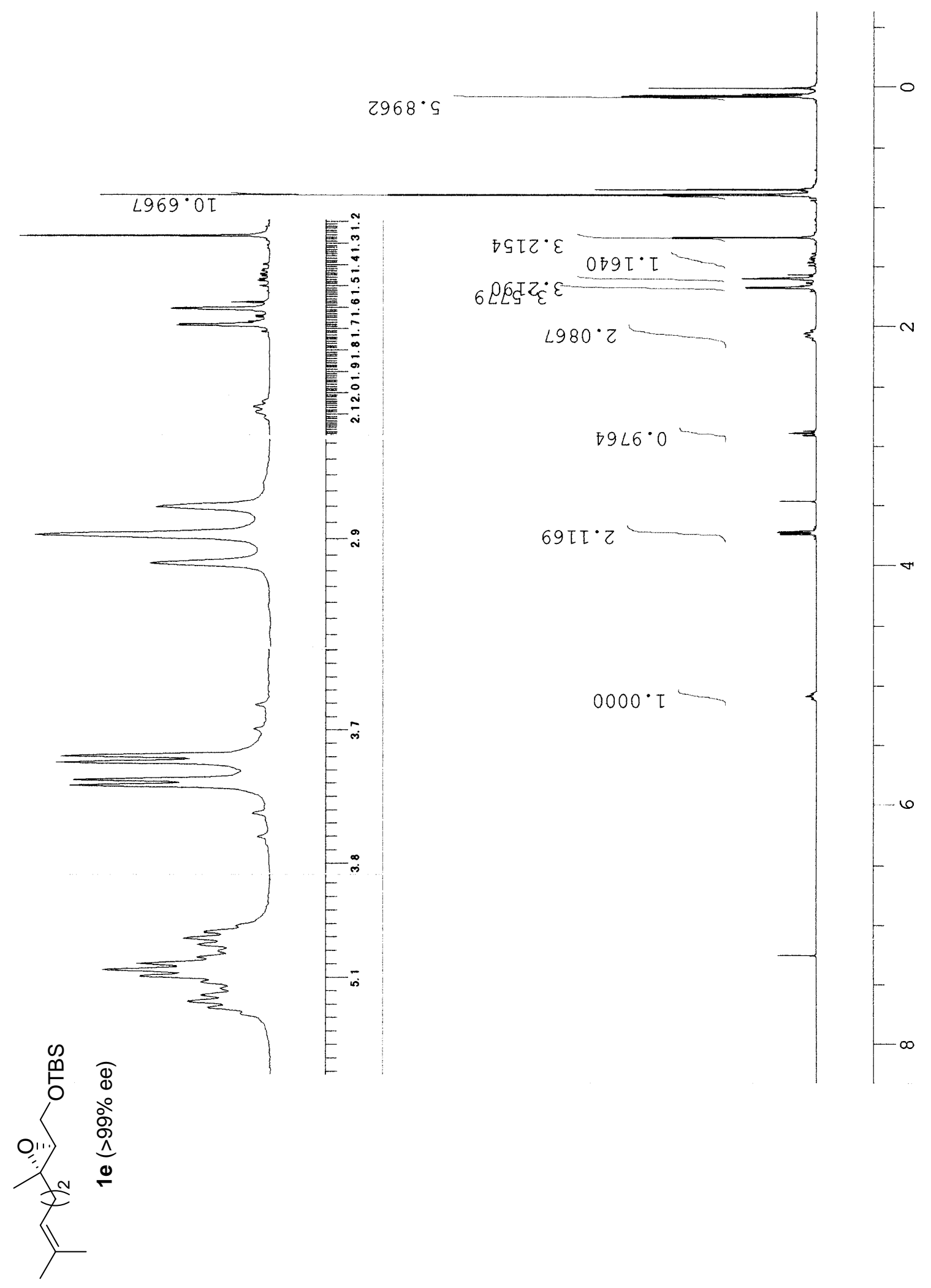




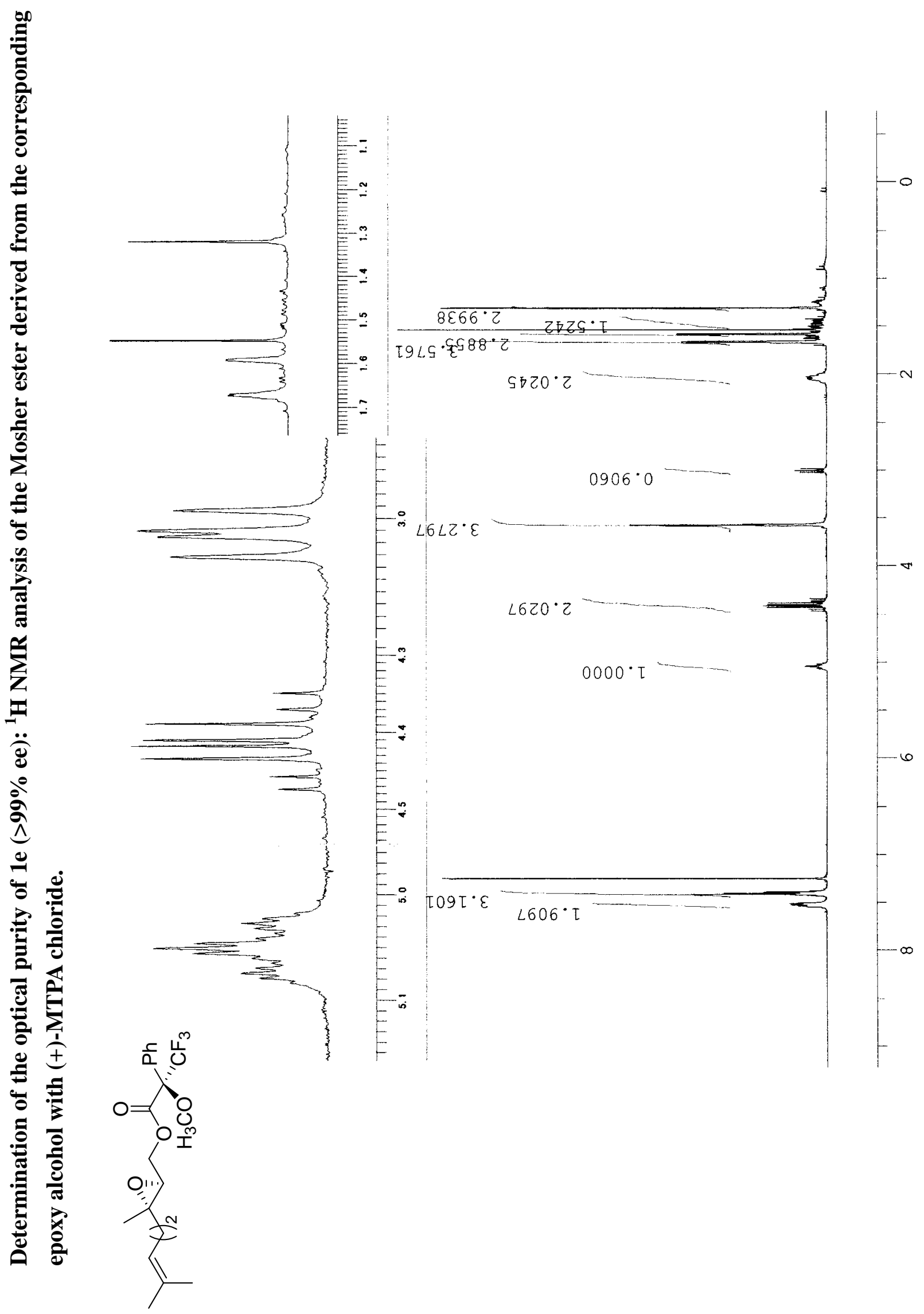




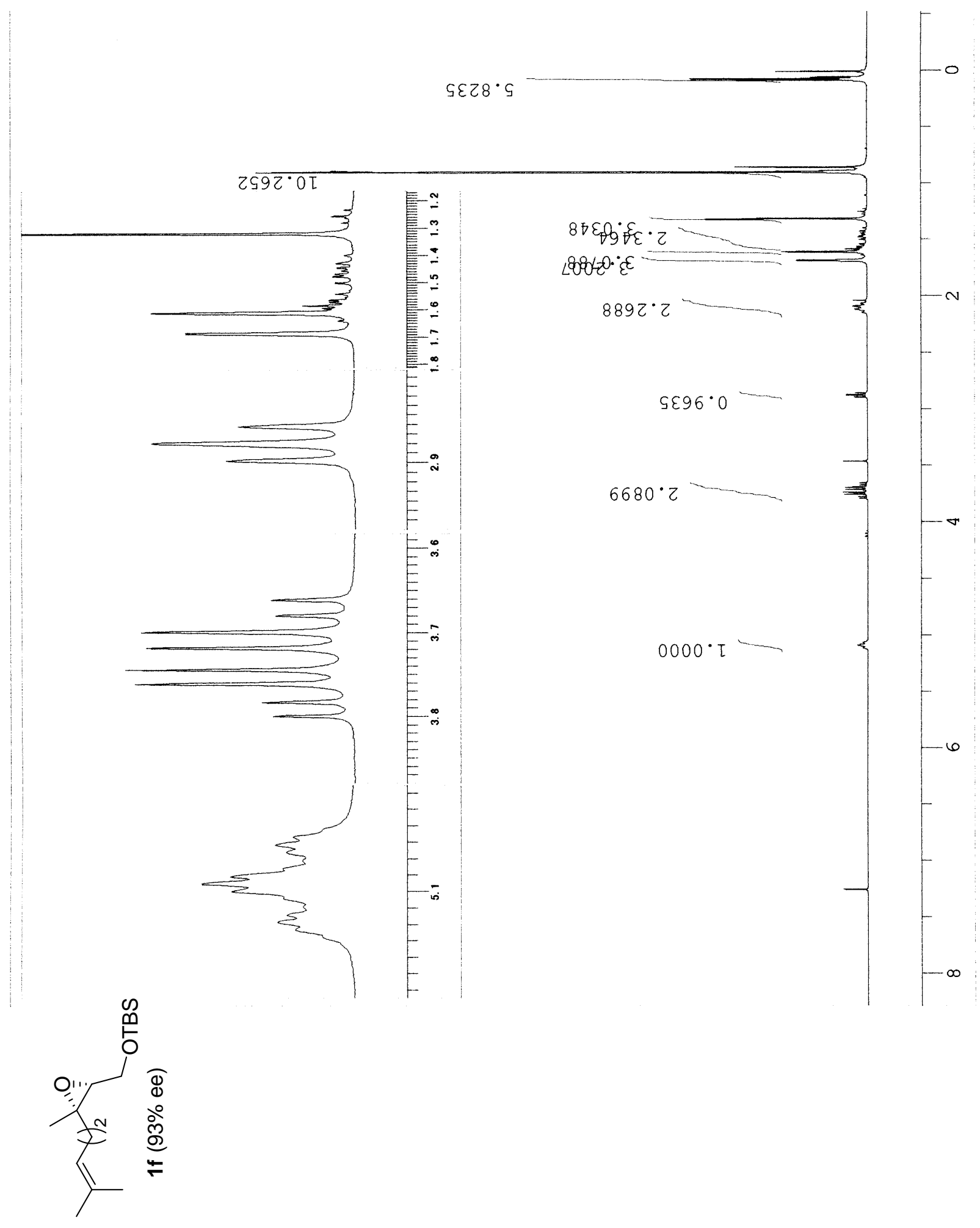




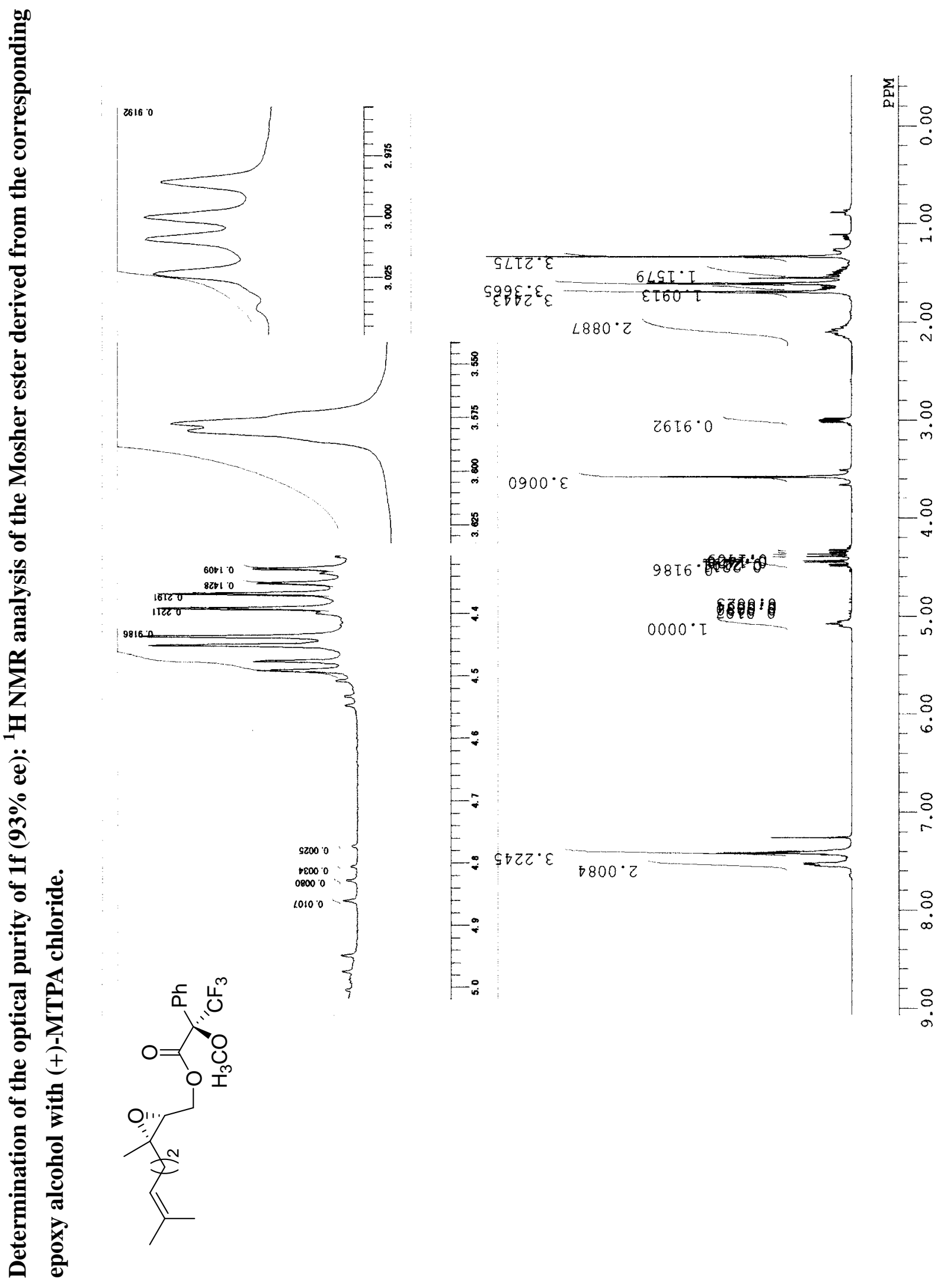



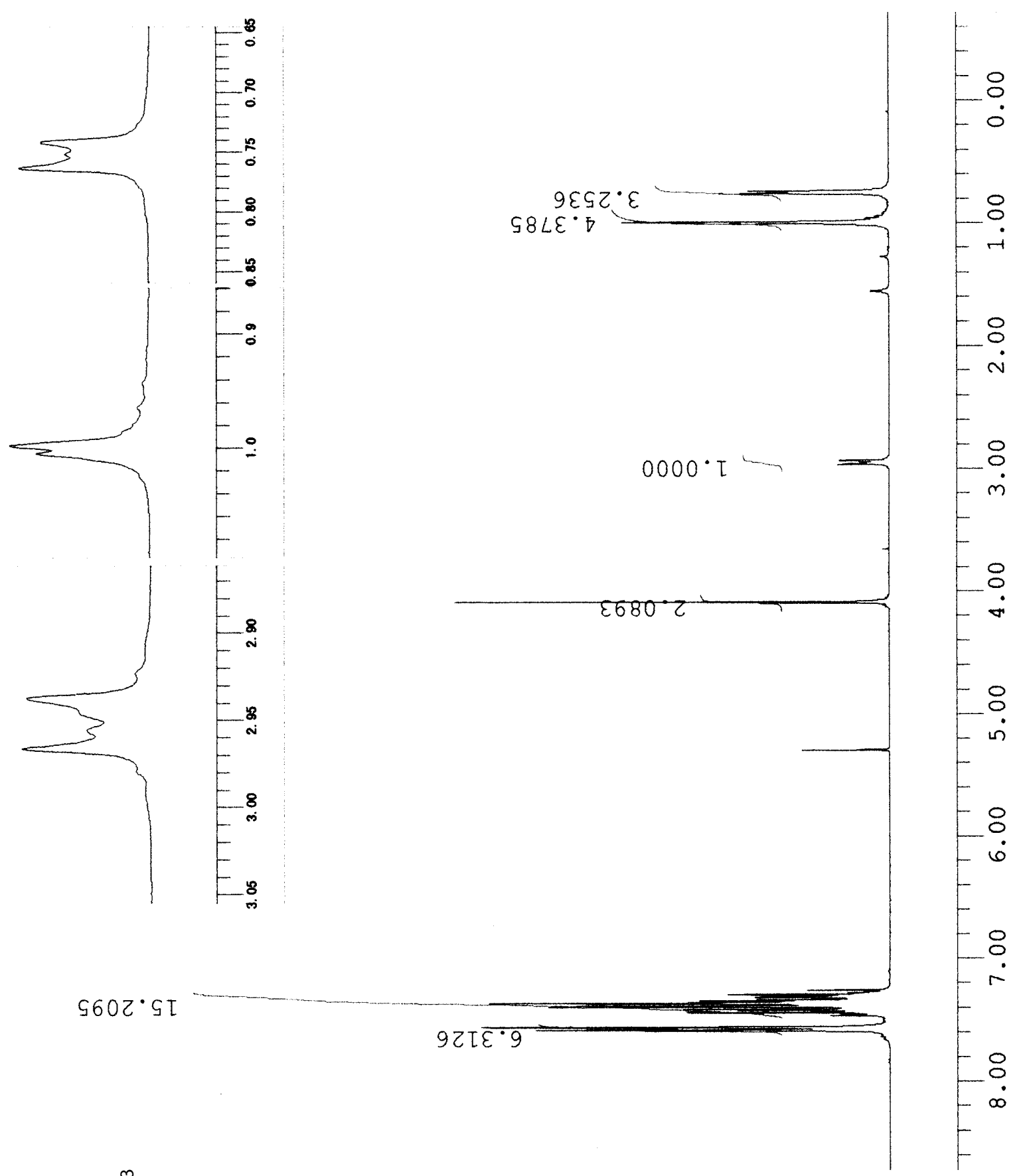

$\underbrace{1}_{\frac{1}{\alpha}}$ 


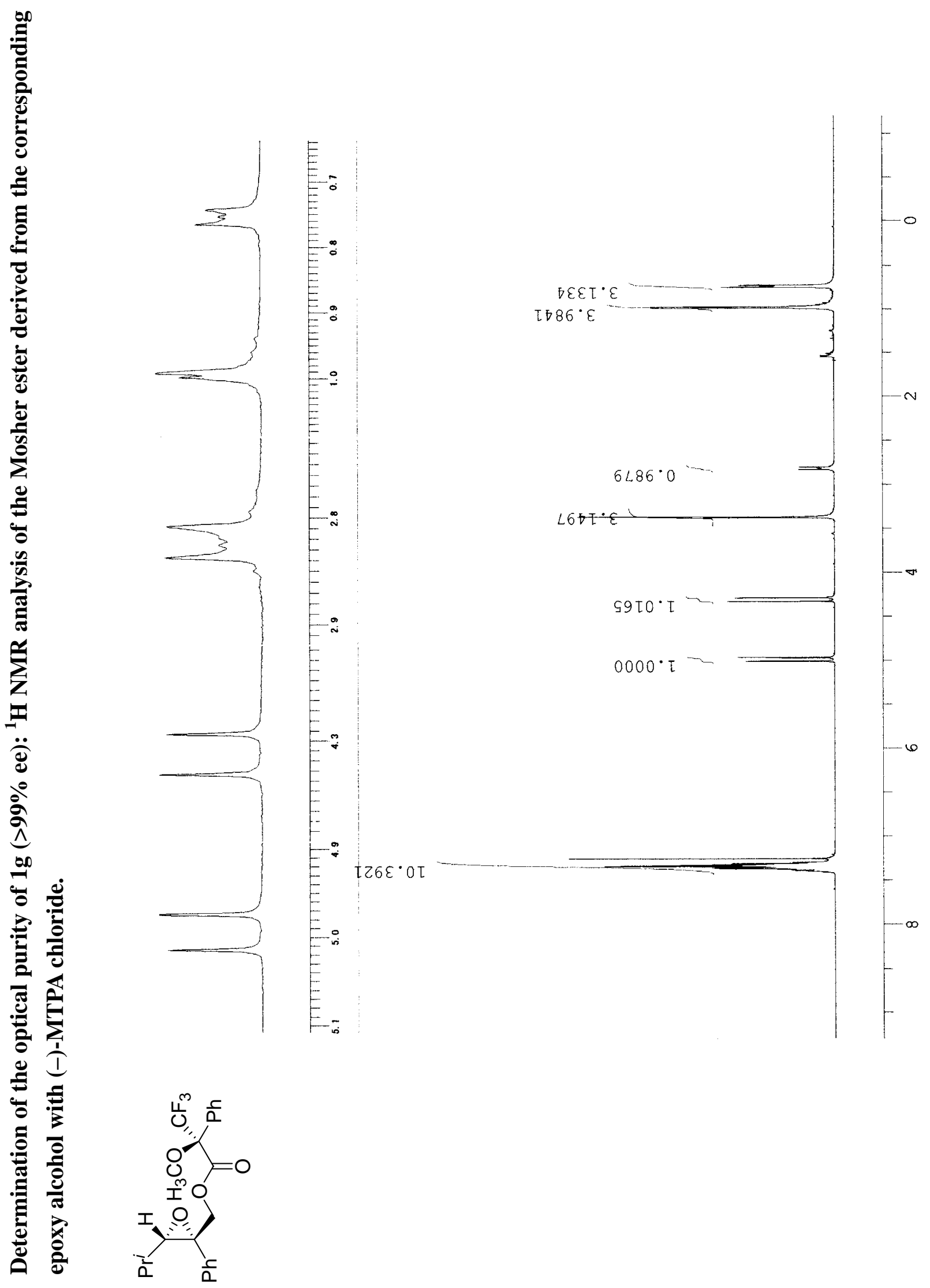




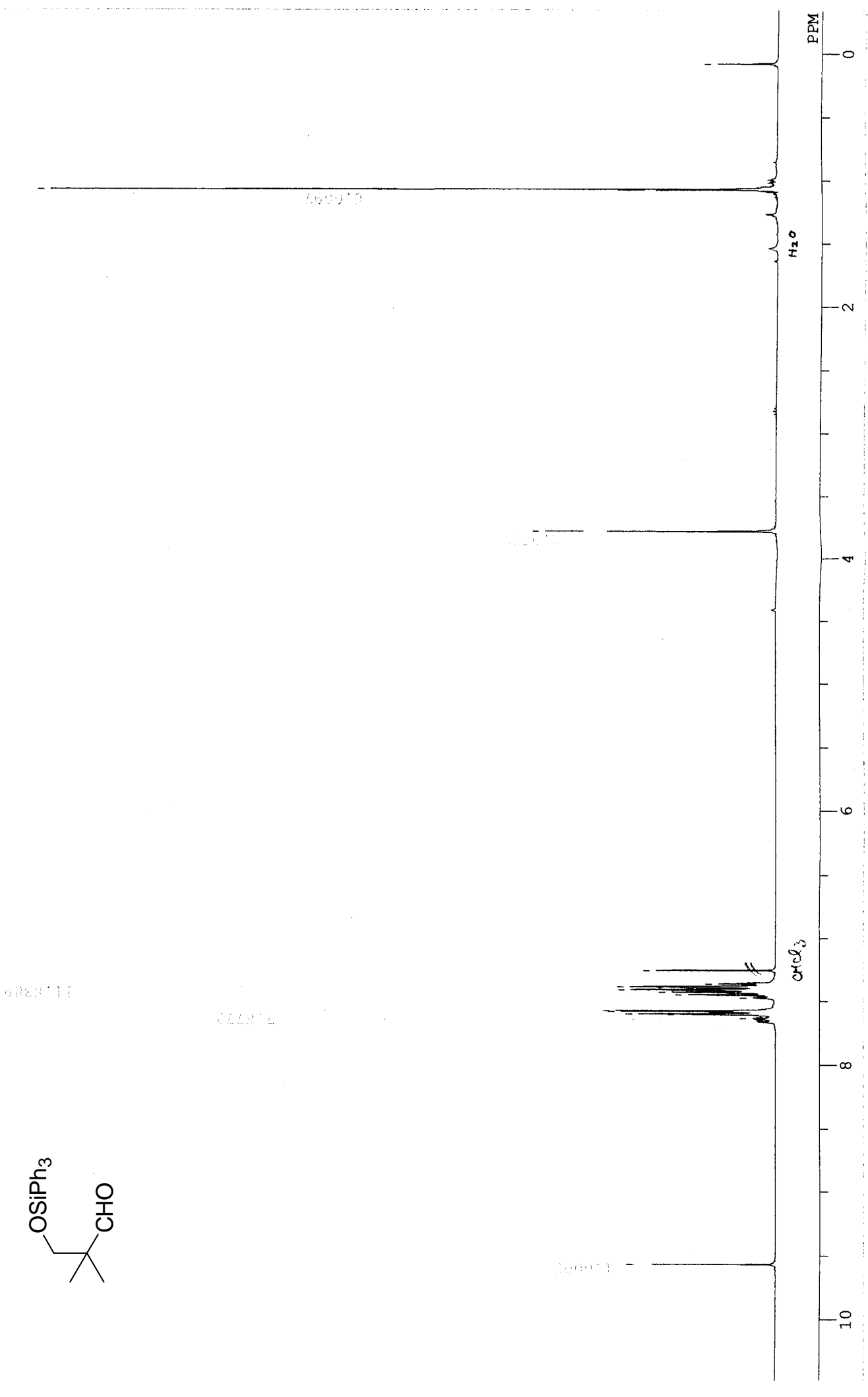




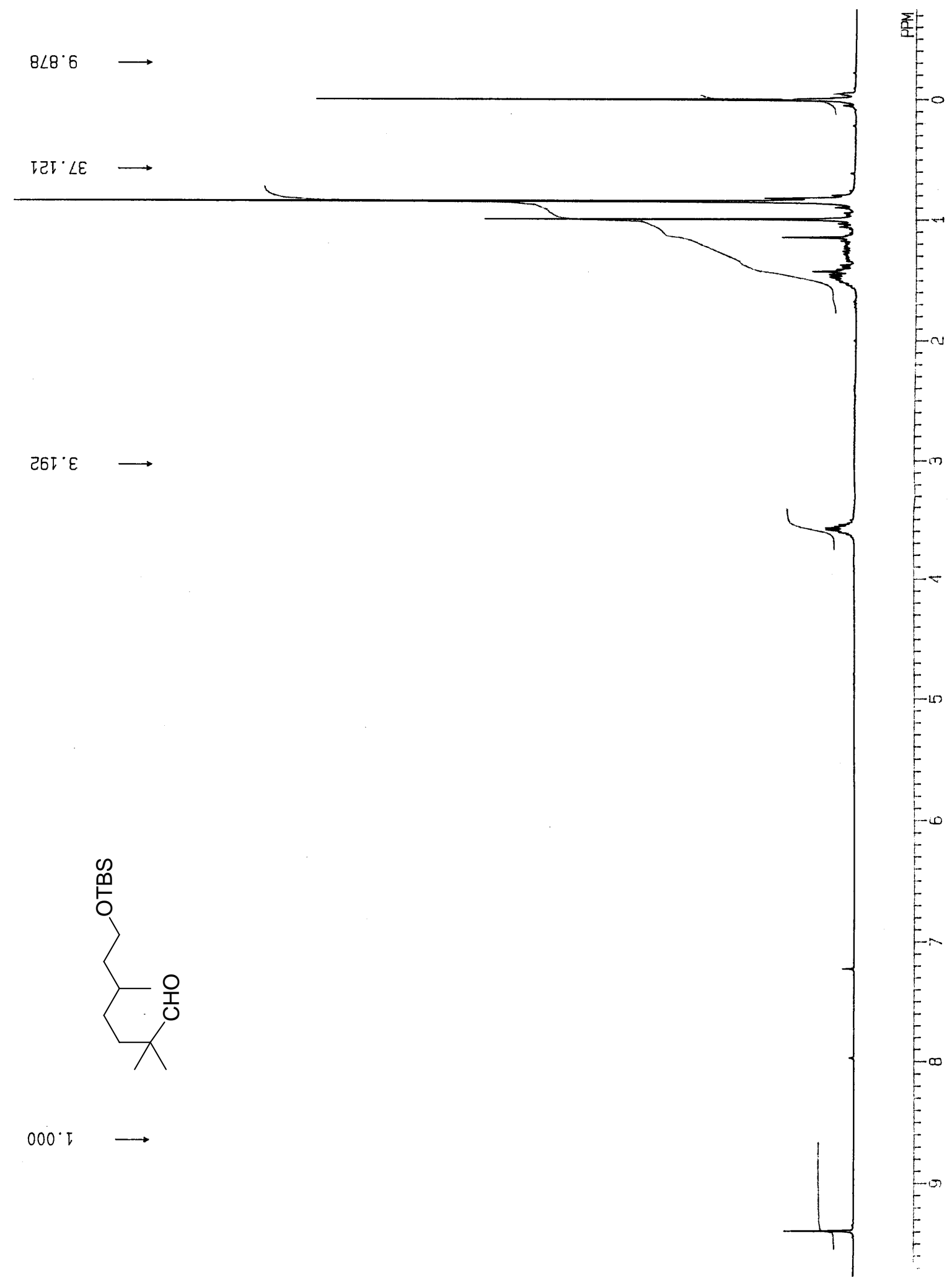




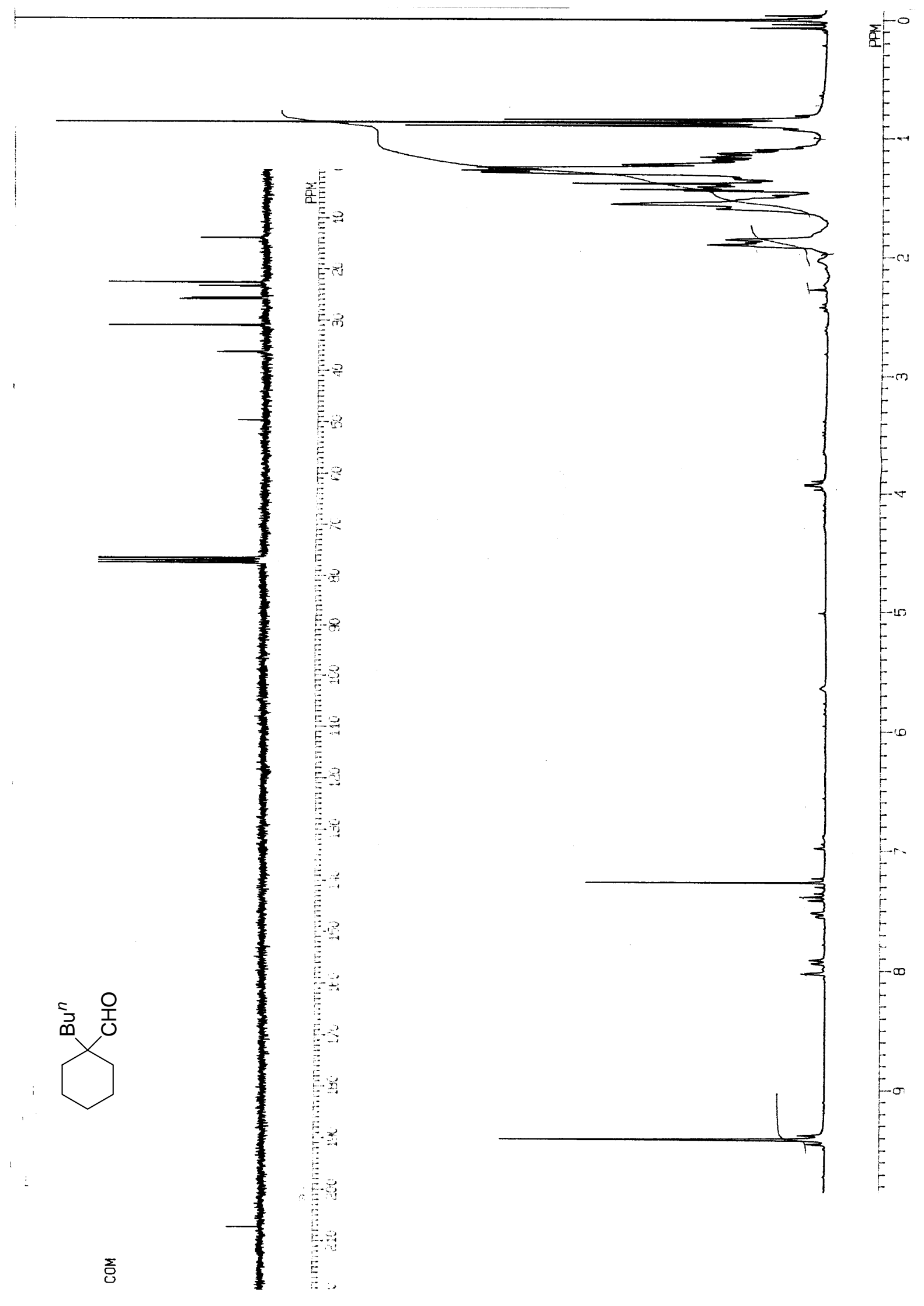




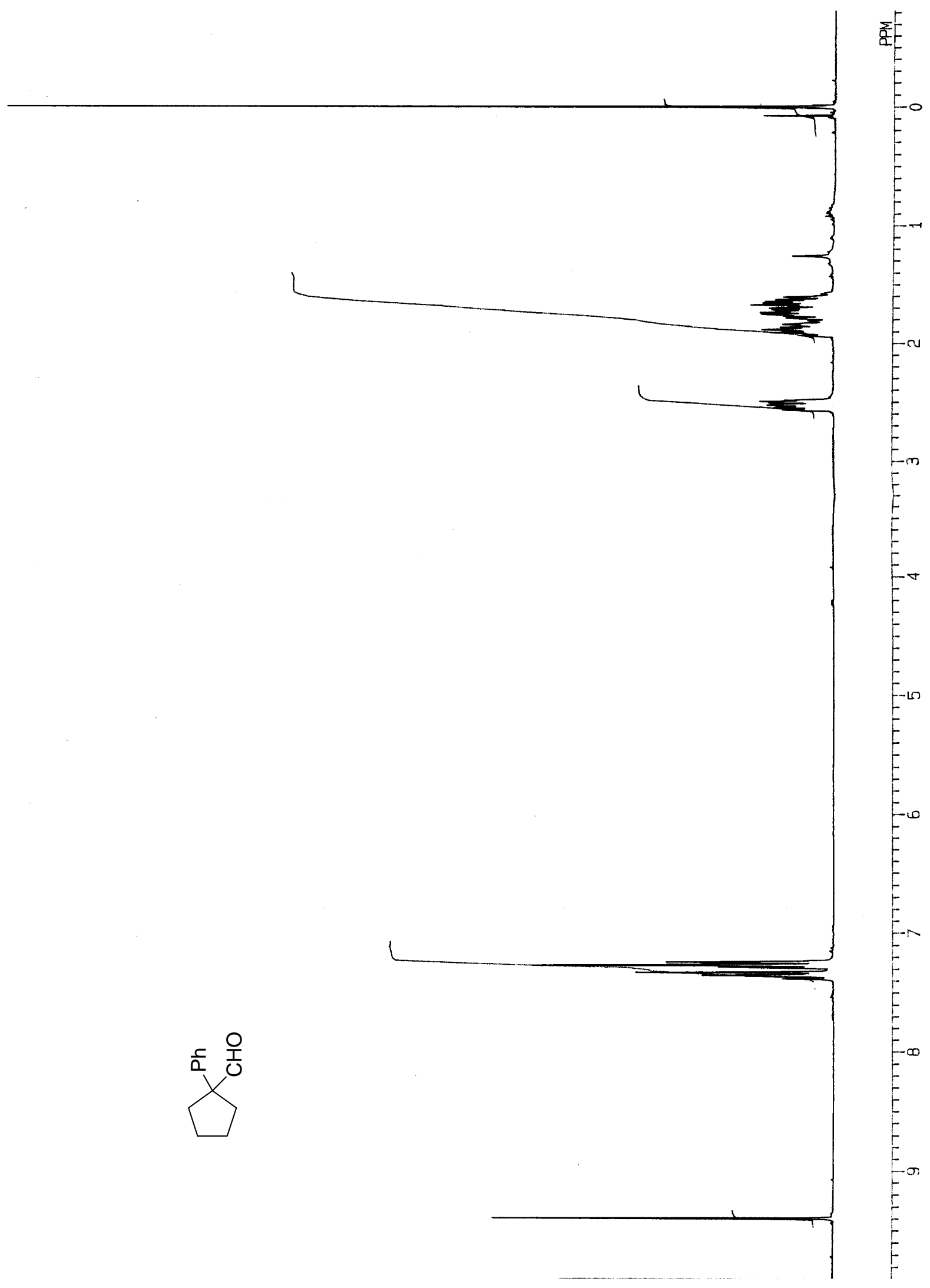




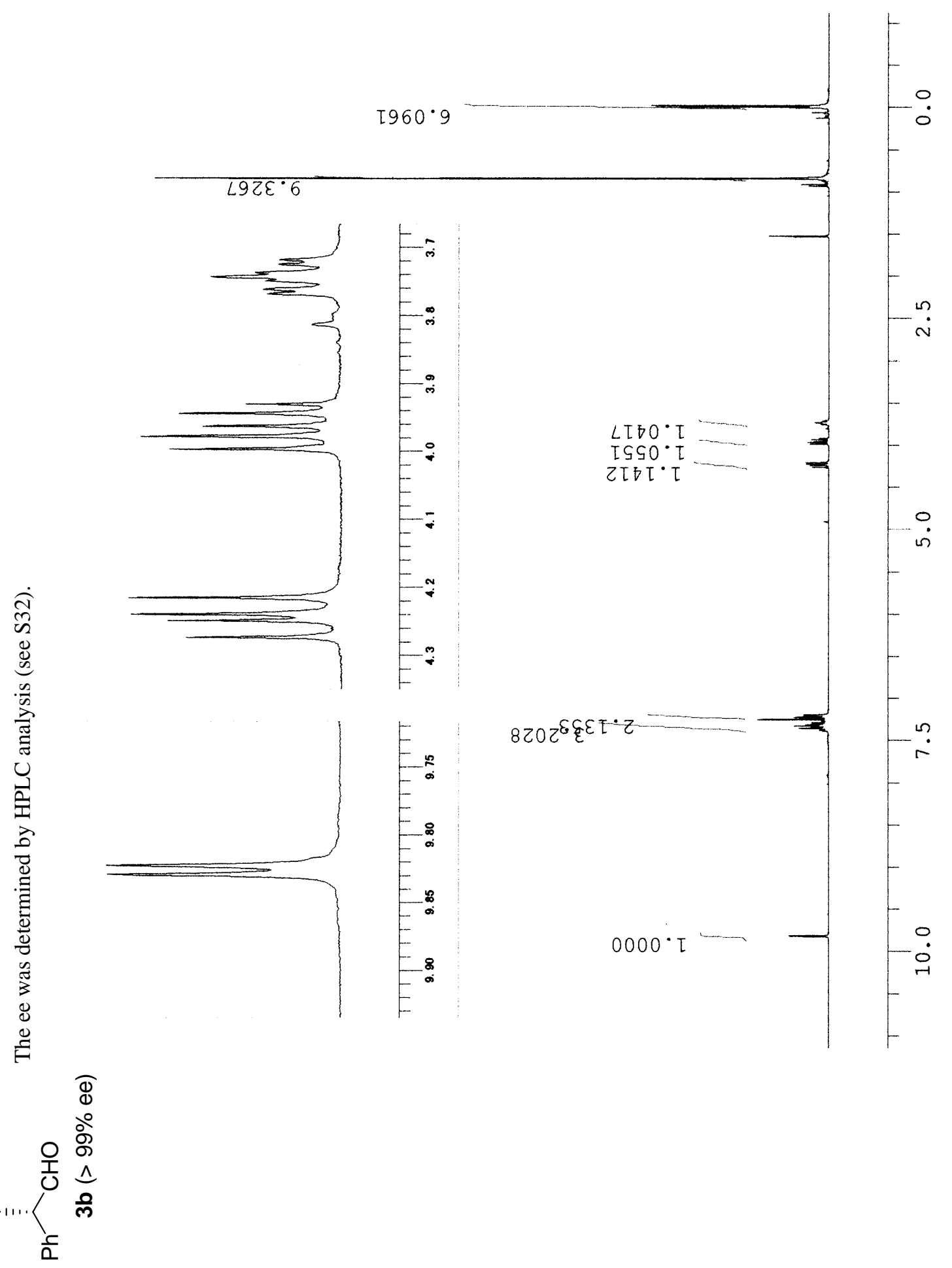




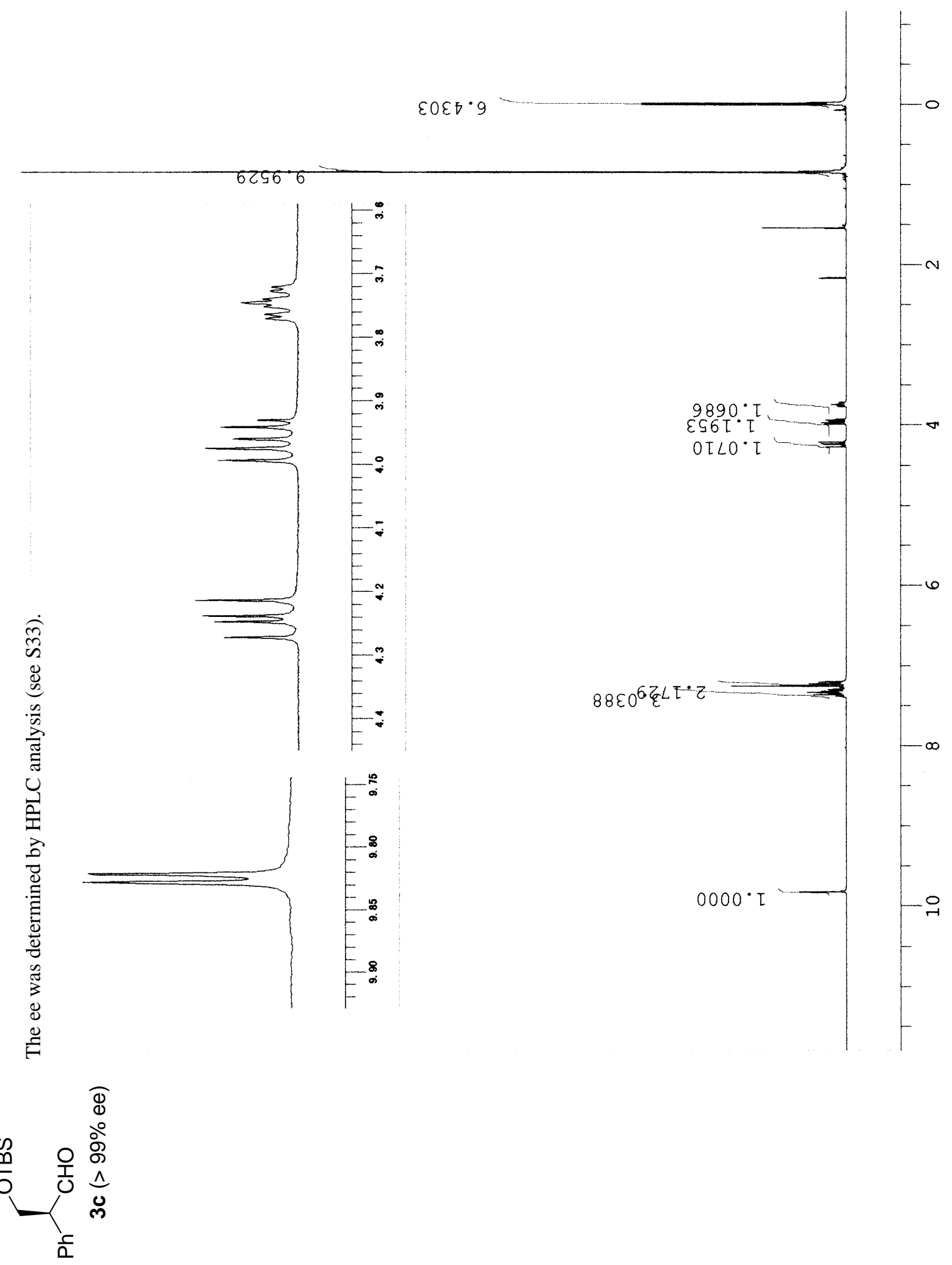




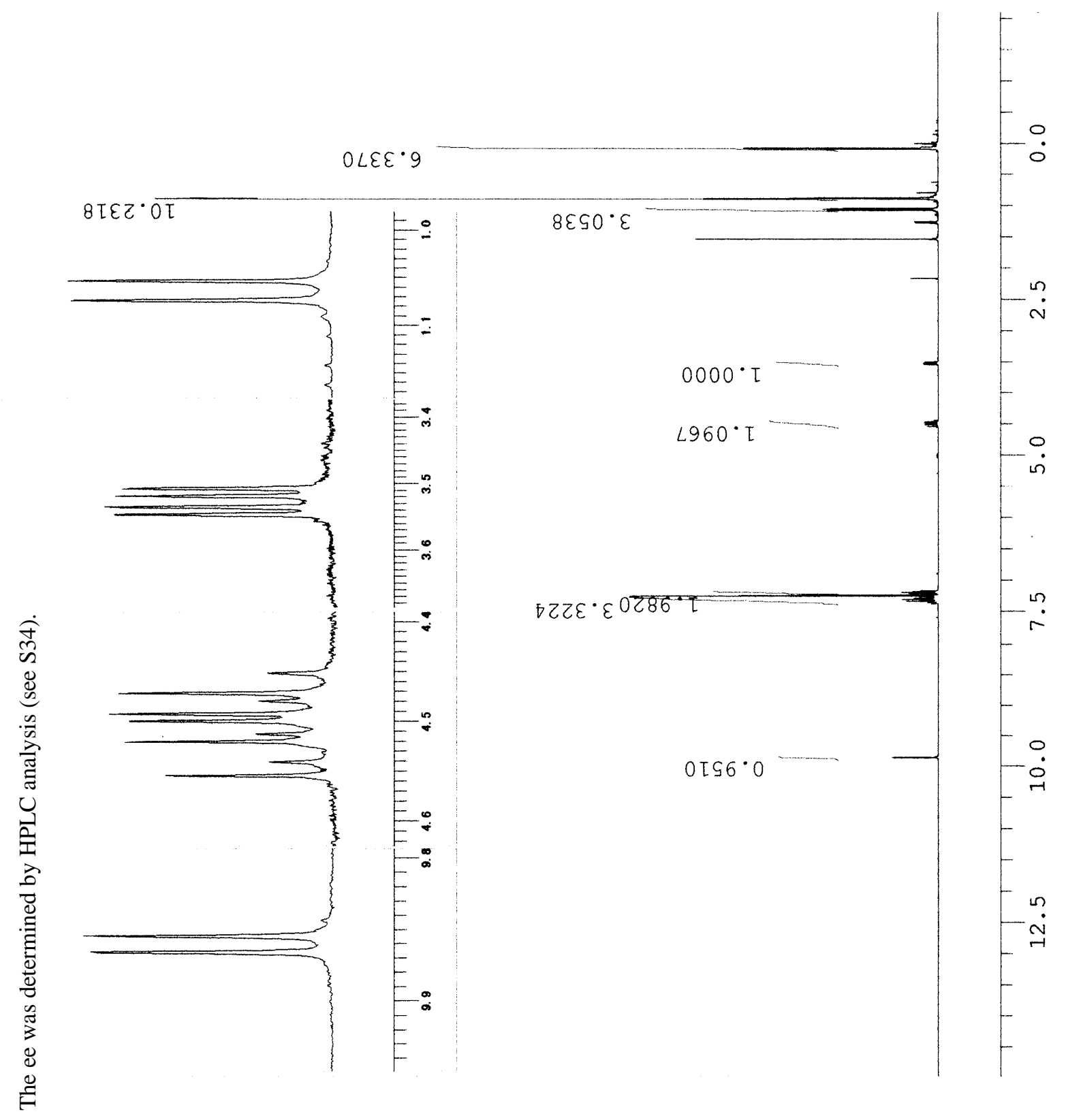

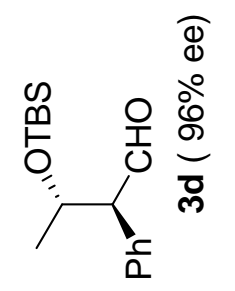




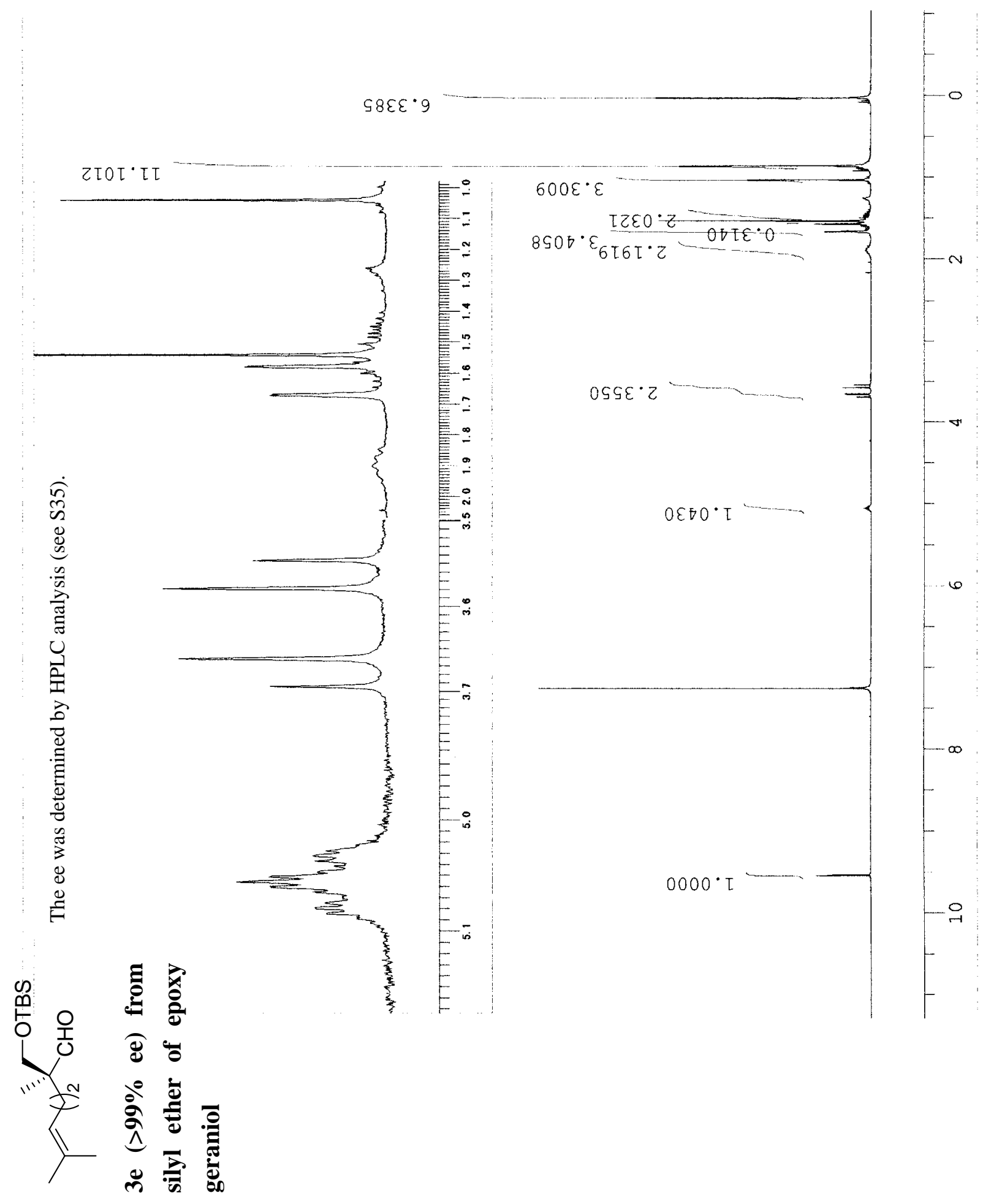




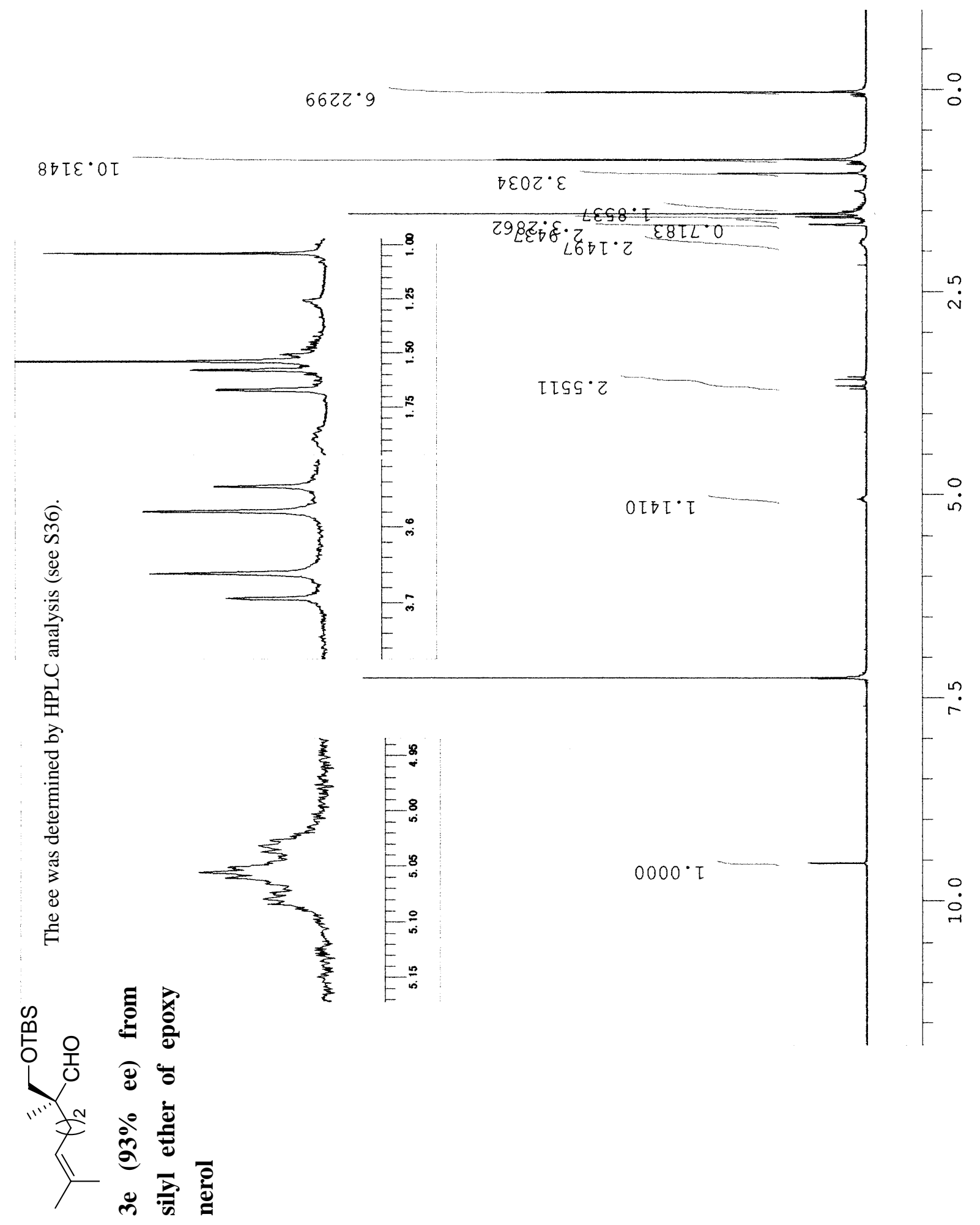




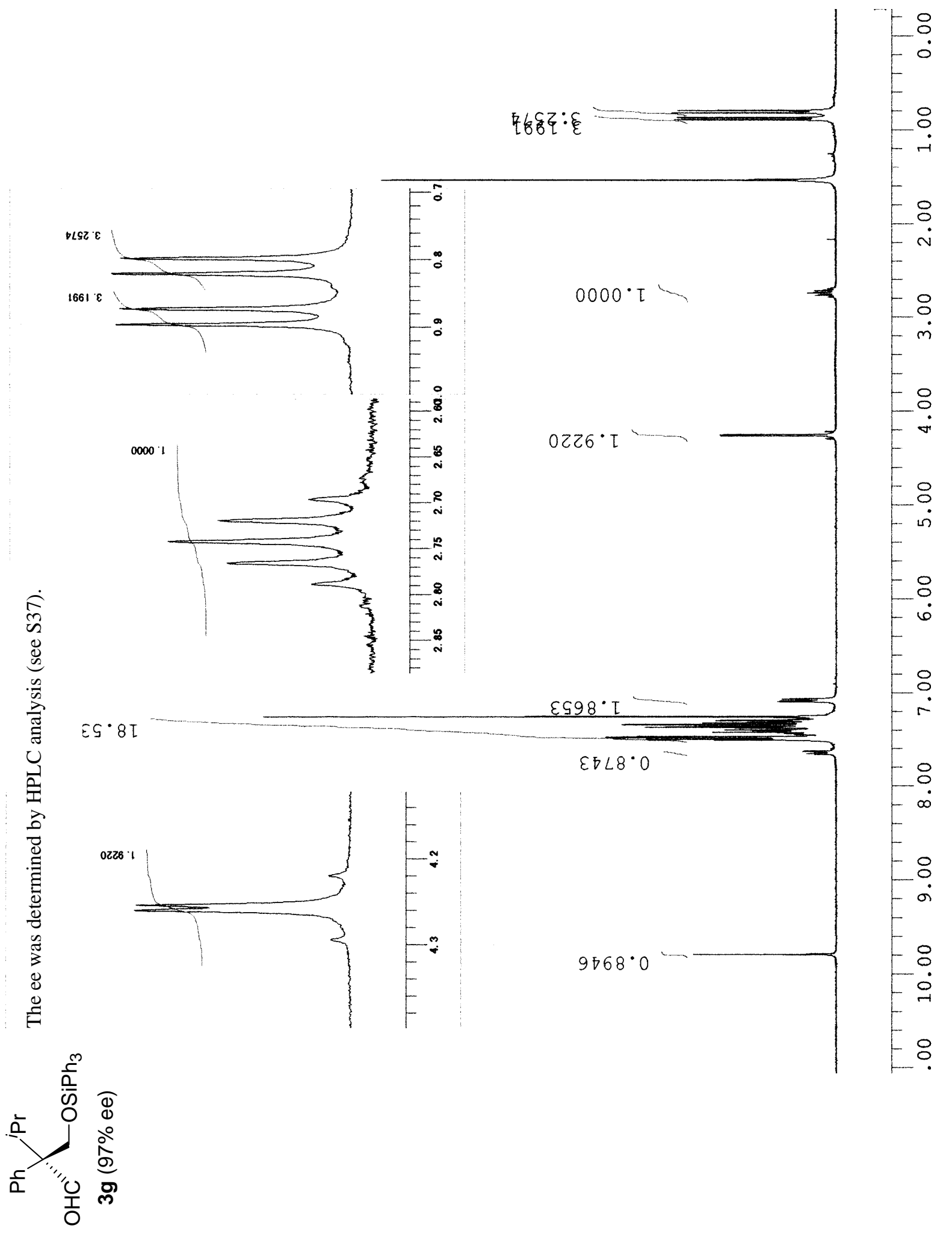


Determination of the optical purity of $1 \mathrm{~d}(96 \% \mathrm{ee})$.

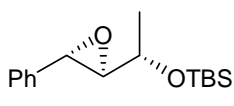

1d

Chiral HPLC: Daicel CHIRALCEL OD-H, 1000:0.3 Hexane:2-Propanol, 0.5 mL/min, $220 \mathrm{~nm}$

racemic (erythro >99\%)

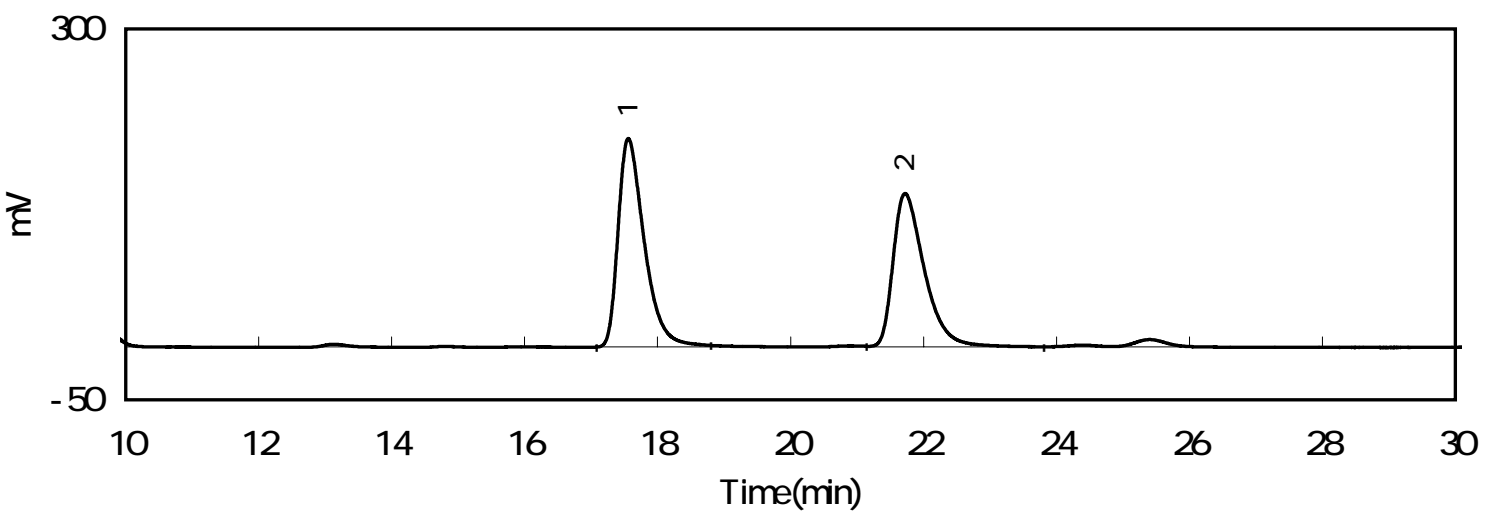

\begin{tabular}{|c|c|c|c|}
\hline No. & $\mathrm{Rt}(\mathrm{min})$ & Area & Area Percent \\
\hline 1 & 17.56 & 5078678.6 & 50.779 \\
\hline & 21.72 & 4922756.2 & 49.220 \\
\hline total & & 10001435 & $\overline{10}$ \\
\hline
\end{tabular}

$96 \% e e$

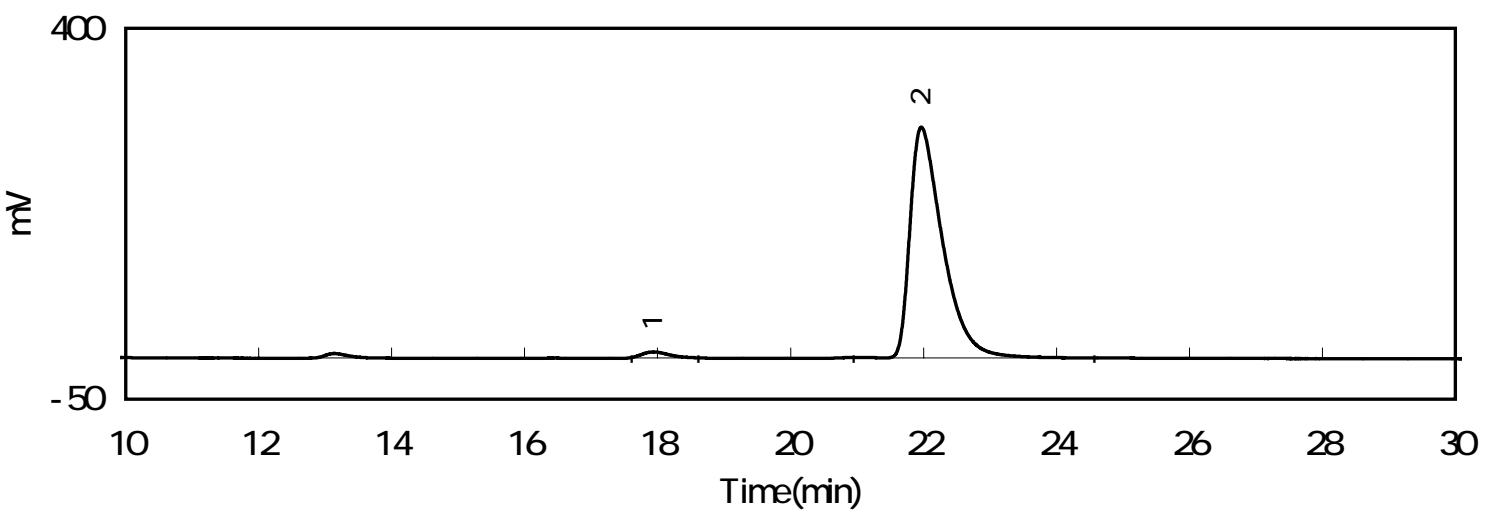

\begin{tabular}{|c|c|c|c|}
\hline No. & $\mathrm{Rt}$ (min) & Area & Area Percent \\
\hline 1 & 17.93 & 195193.4 & 2.0069 \\
\hline 2 & 21.97 & 9530802.4 & 97.9931 \\
\hline total & & 9725995.8 & 100 \\
\hline
\end{tabular}


Determination of the optical purity of $3 \mathrm{~b}$ (>99\% ee).

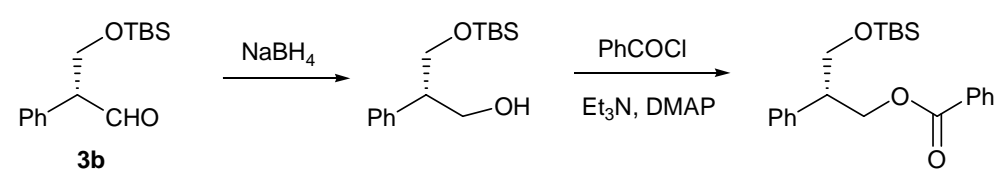

Chiral HPLC: Daicel CHIRALPAK AD-H, 500:1 Hexane:2-Propanol, $0.5 \mathrm{~mL} / \mathrm{min}, 220 \mathrm{~nm}$

racemic

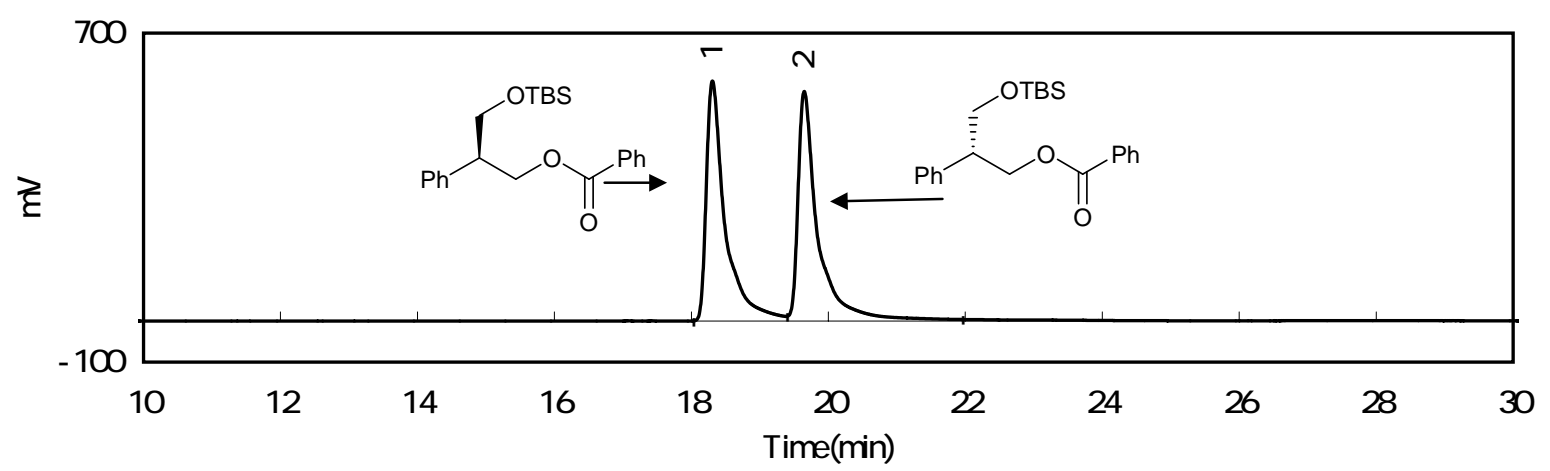

\begin{tabular}{|c|c|c|c|}
\hline No. & $\mathrm{Rt}(\mathrm{min})$ & Area & Area Percent \\
\hline 1 & 18.31 & 11144141.4 & 50.2385 \\
\hline 2 & 19.65 & 11038345.8 & 49.7615 \\
\hline total & & 22182487.2 & 100 \\
\hline
\end{tabular}

$>99 \% e e$

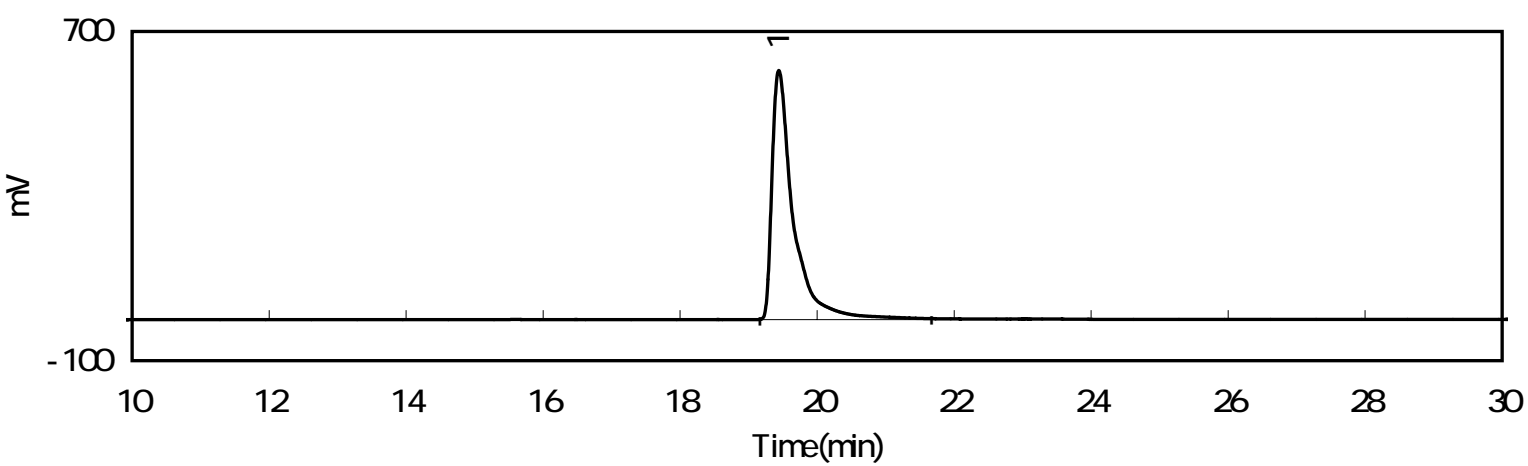

\begin{tabular}{|c|c|c|c|}
\hline No. & $\mathrm{Rt}(\mathrm{min})$ & Area & Area Percent \\
\hline 1 & 19.44 & 12979321 & 100 \\
\hline total & & 12979321 & 100 \\
\hline
\end{tabular}


Determination of the optical purity of $3 \mathrm{c}(>99 \% \mathrm{ee})$.

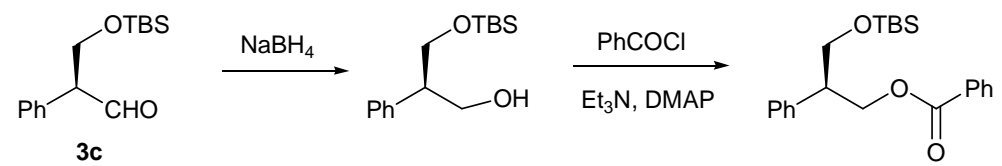

Chiral HPLC: Daicel CHIRALPAK AD-H, 500:1 Hexane:2-Propanol, $0.5 \mathrm{~mL} / \mathrm{min}, 220 \mathrm{~nm}$

racemic

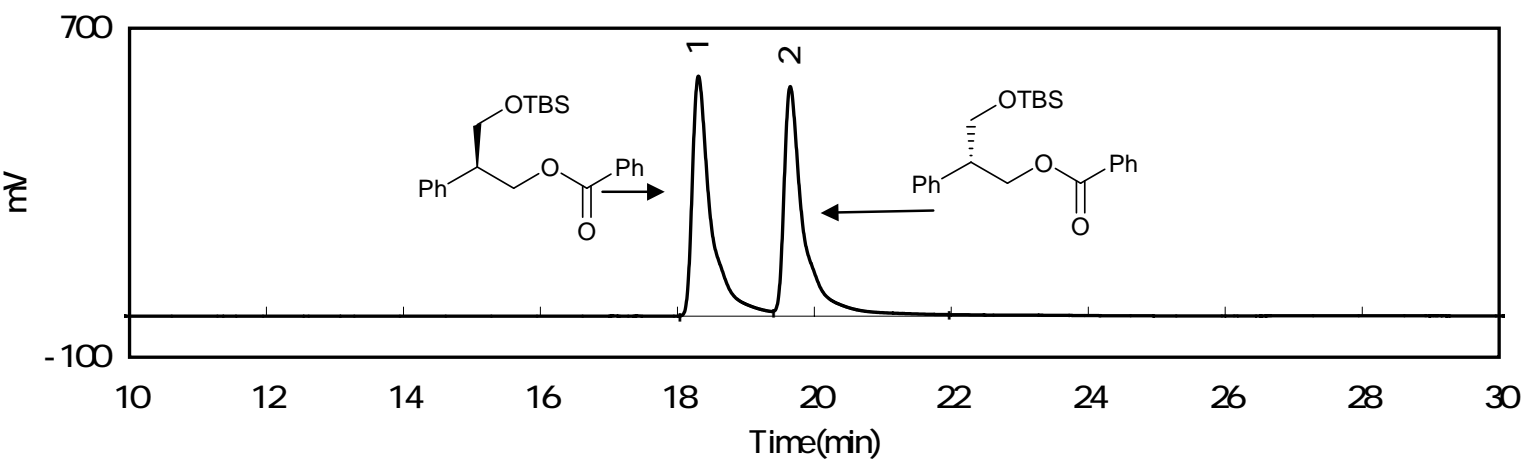

\begin{tabular}{|c|c|c|c|}
\hline No. & Rt (min) & Area & Area Percent \\
\hline 1 & 18.31 & 11144141.4 & 50.2385 \\
\hline 2 & 19.65 & 11038345.8 & 49.7615 \\
\hline total & & 22182487.2 & 100 \\
\hline
\end{tabular}

$>99 \% e e$

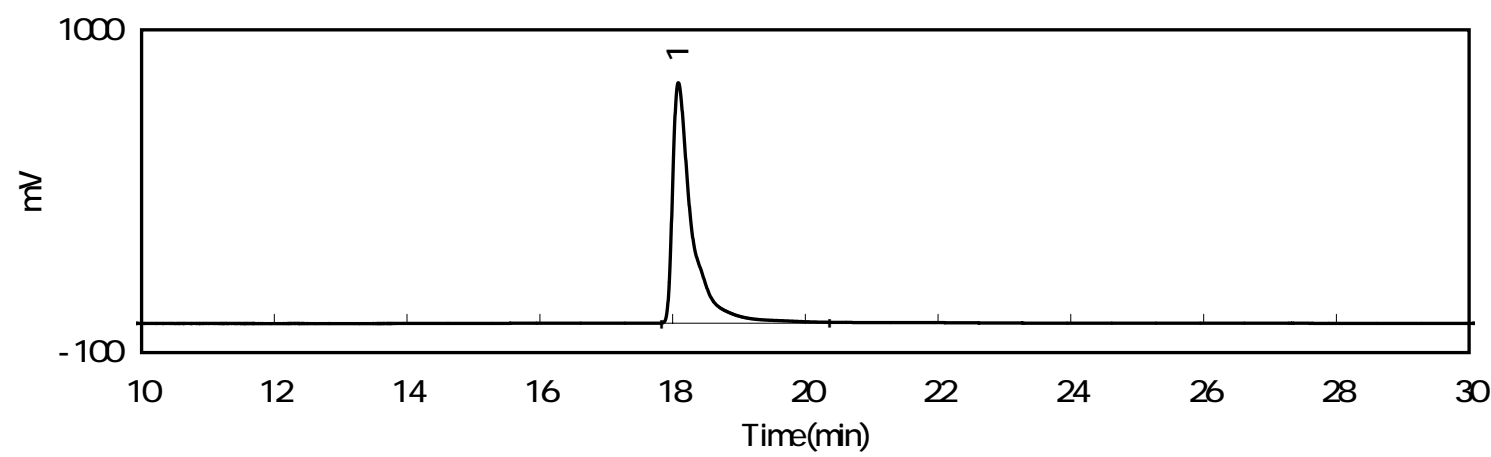

\begin{tabular}{|c|c|c|c|}
\hline No. & Rt (min) & Area & Area Percent \\
\hline 1 & 18.09 & 16672528.4 & 100 \\
\hline total & & 16672528.4 & 100 \\
\hline
\end{tabular}


Determination of the optical purity of $3 d(96 \%$ ee).

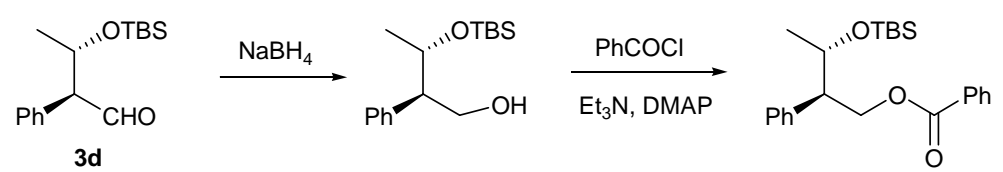

Chiral HPLC: Daicel CHIRALCEL OD-H, 500:0.2 Hexane:2-Propanol, 0.5 mL/min, $220 \mathrm{~nm}$

racemic (65:35 erythro:threo mixture)

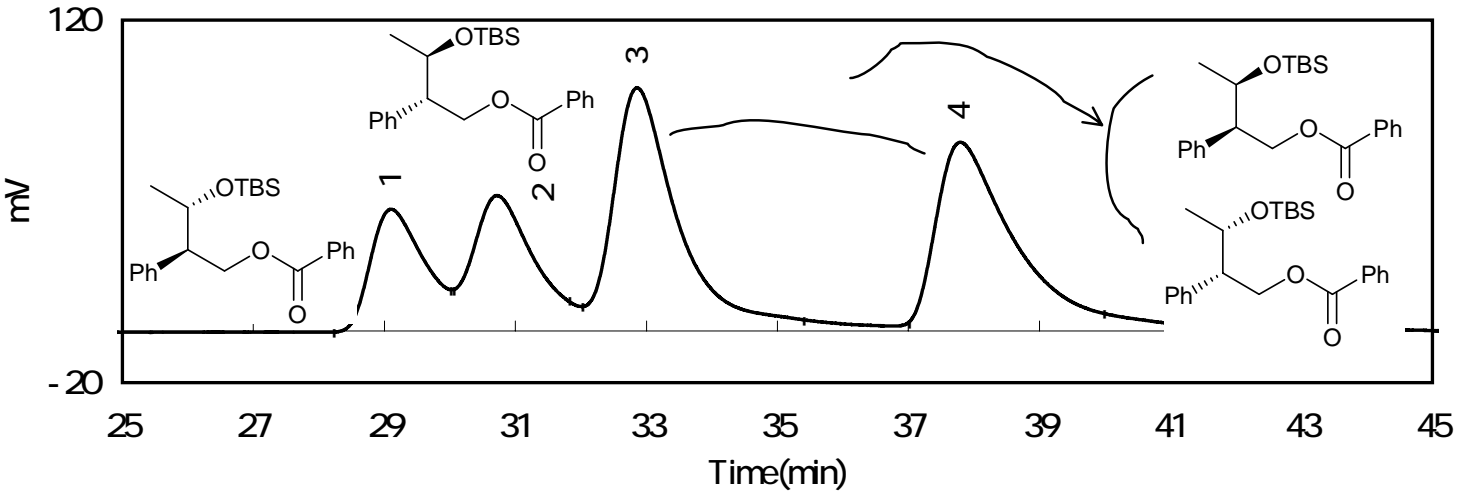

\begin{tabular}{|r|r|r|r|}
\hline No. & Rt $(\mathrm{min})$ & Area & Area Percent \\
\hline 1 & 29.1 & 2379737.22 & 17.1213 \\
\hline 2 & 30.73 & 2467469.47 & 17.7525 \\
\hline 3 & 32.86 & 4548955.97 & 32.728 \\
\hline 4 & 37.79 & 4503116.14 & 32.3982 \\
\hline total & & 13899278.8 & 100 \\
\hline
\end{tabular}

$96 \% e e$

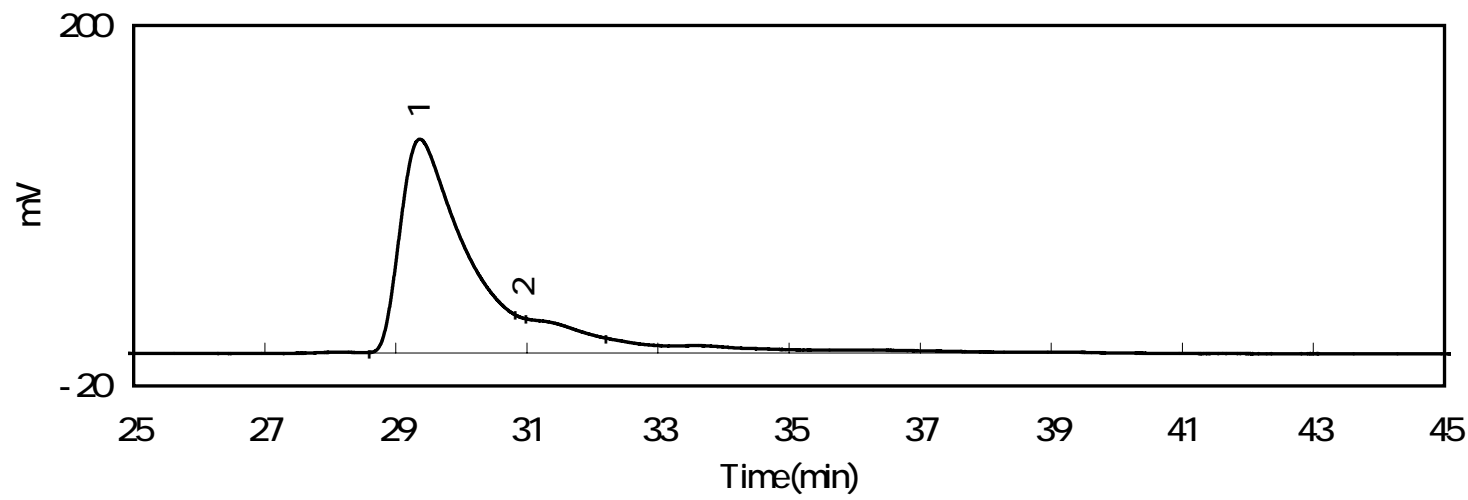

\begin{tabular}{|l|r|r|r|}
\hline No. & Rt (min) & \multicolumn{1}{|l|}{ Area } & Area Percent \\
\hline 1 & 29.36 & 6937901.4 & 98.2005 \\
\hline 2 & 30.96 & 127135.3 & 1.7995 \\
\hline total & & 7065036.7 & 100 \\
\hline
\end{tabular}


Determination of the optical purity of $3 \mathrm{e}(>99 \%$ ee) prepared from silyl ether of epoxy geraniol.
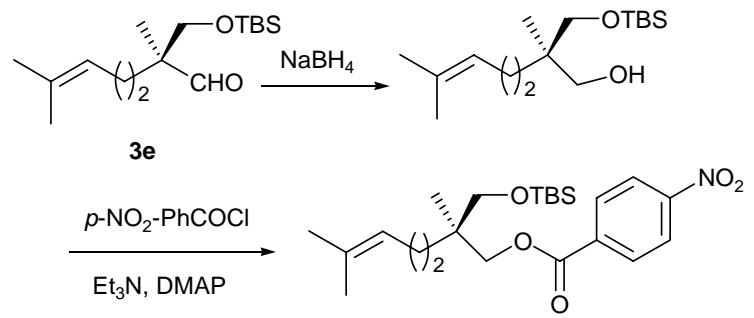

Chiral HPLC: Daicel CHIRALCEL OD-H, 500:1 Hexane:2-Propanol, 0.4 mL/min, $250 \mathrm{~nm}$

\section{racemic}

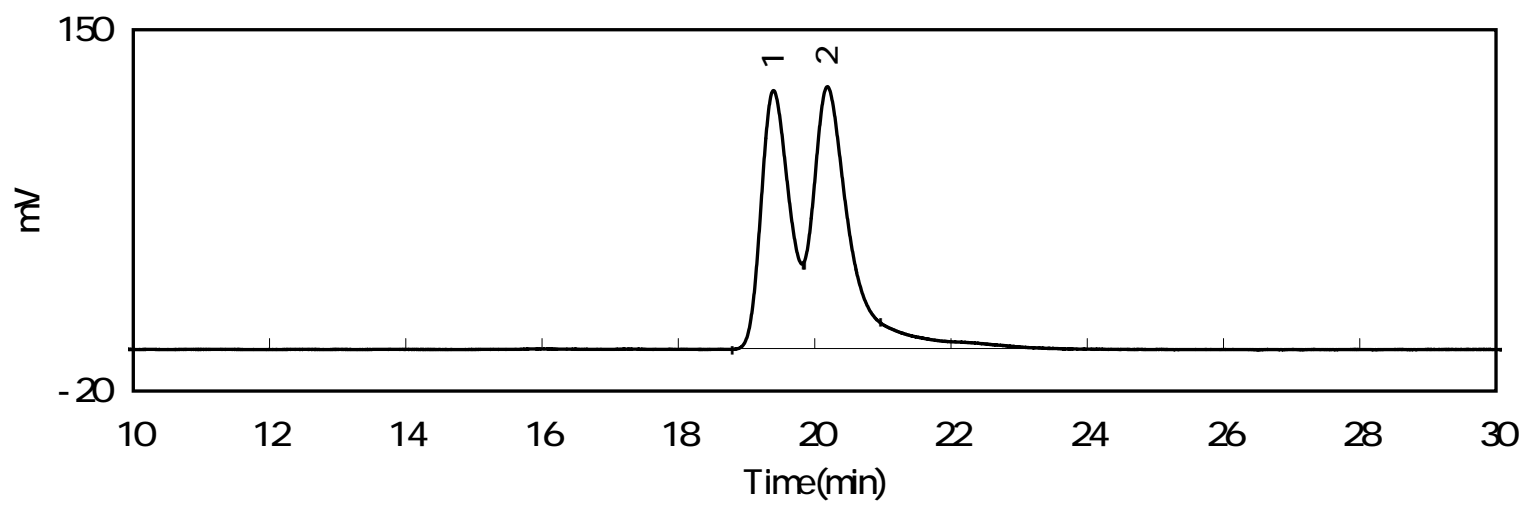

\begin{tabular}{|c|c|c|c|}
\hline No. & $\mathrm{Rt}(\mathrm{min})$ & Area & Area Percent \\
\hline 1 & 19.4 & 2425742.45 & 49.2108 \\
\hline 2 & 20.19 & 2503546.38 & 50.7892 \\
\hline total & & 4929288.8 & 100 \\
\hline
\end{tabular}

$>99 \% e e$

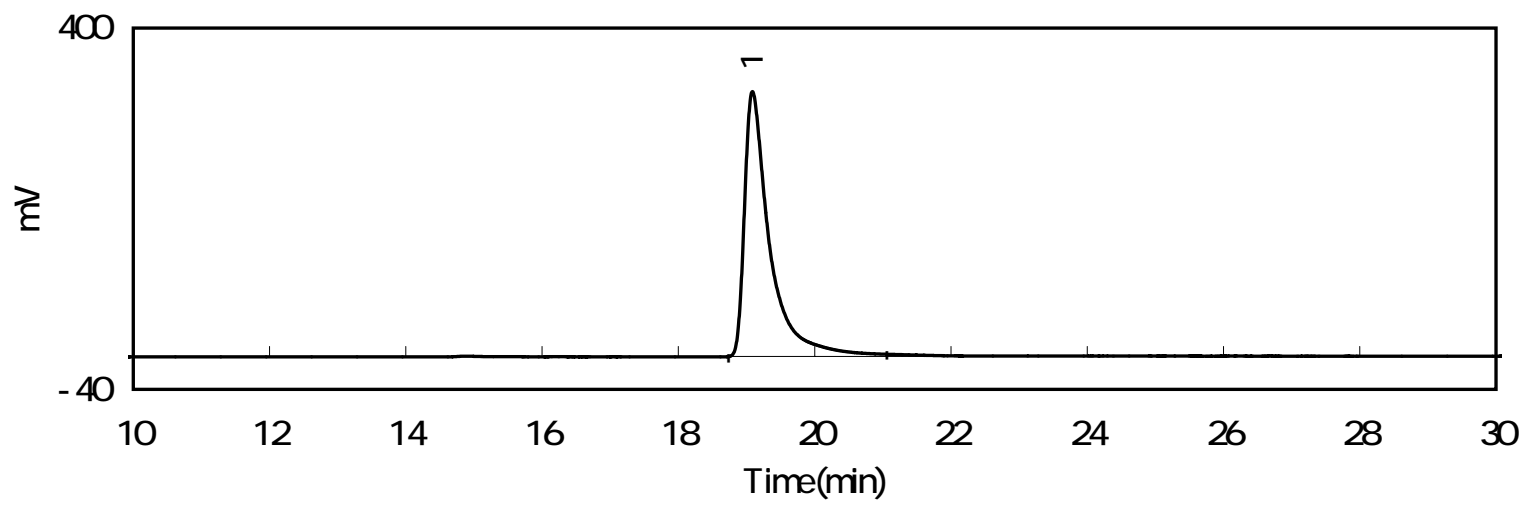

\begin{tabular}{|c|c|c|c|}
\hline No. & $\mathrm{Rt}(\mathrm{min})$ & Area & Area Percent \\
\hline$\overline{1}$ & 19.09 & 8171320.2 & 322505 \\
\hline total & & 8171320.2 & 32250 \\
\hline
\end{tabular}


Determination of the optical purity of $3 \mathrm{e}(93 \%$ ee) prepared from silyl ether of epoxy nerol.
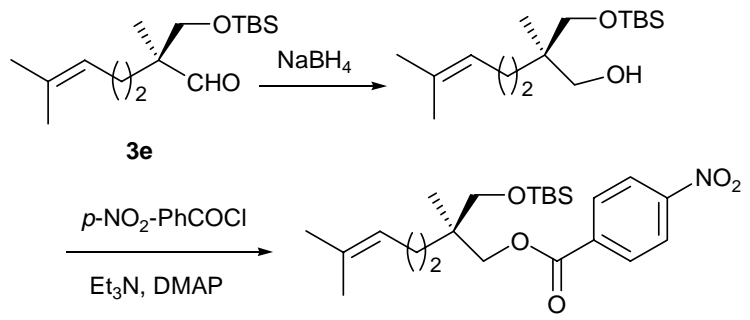

Chiral HPLC: Daicel CHIRALCEL OD-H, 500:1 Hexane:2-Propanol, $0.4 \mathrm{~mL} / \mathrm{min}, 250 \mathrm{~nm}$

\section{racemic}

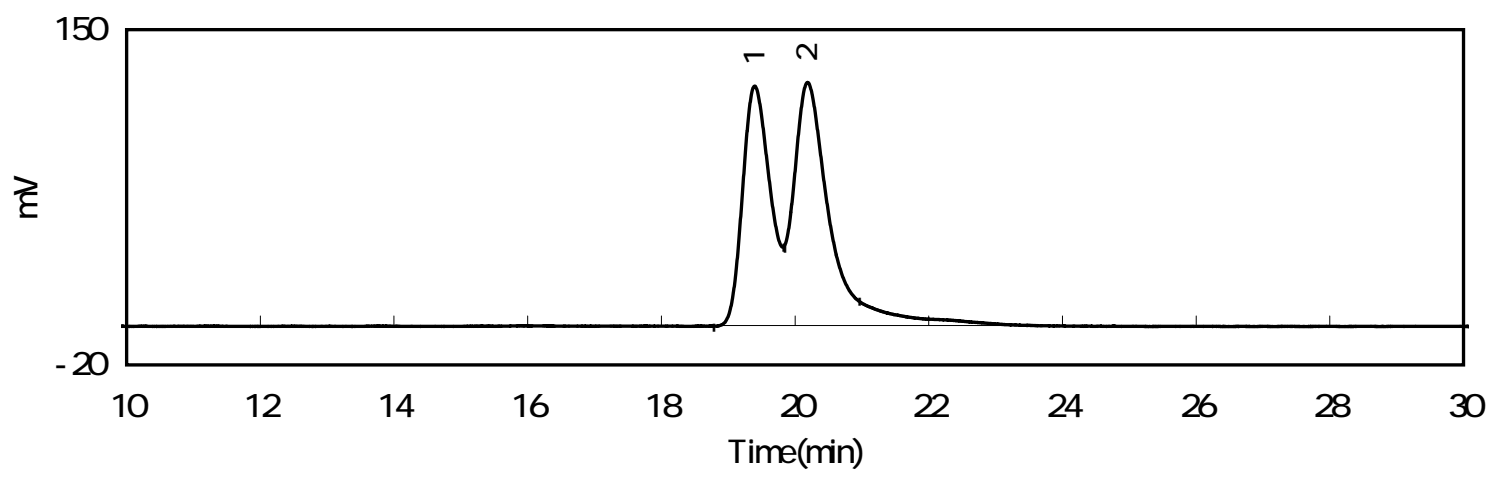

\begin{tabular}{|c|c|c|c|}
\hline No. & $\mathrm{Rt}(\mathrm{min})$ & Area & Area Percent \\
\hline 1 & 19.4 & 2425742.45 & 49.2108 \\
\hline 2 & 20.19 & 2503546.38 & 50.7892 \\
\hline total & & 4929288.8 & 100 \\
\hline
\end{tabular}

$93 \% e e$

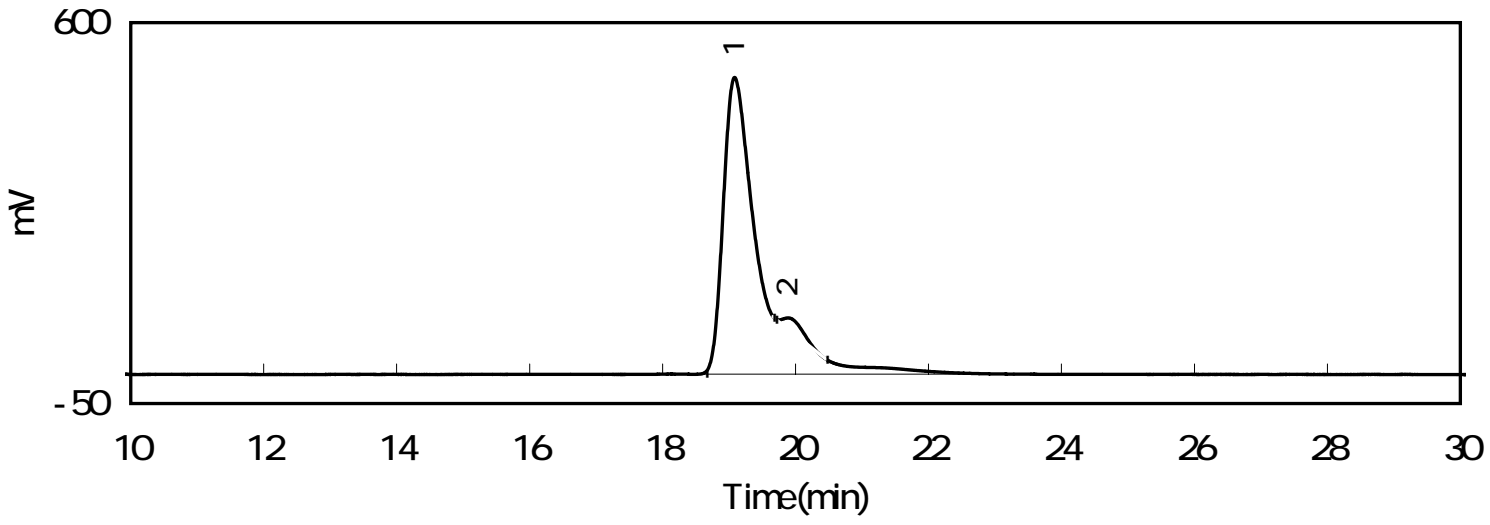

\begin{tabular}{|c|c|c|c|}
\hline No. & $\mathrm{Rt}(\mathrm{min})$ & Area & Area Percent \\
\hline$\overline{1}$ & 19.08 & 12290710.8 & 96.3908 \\
\hline 2 & 19.89 & 460207.6 & 3.6092 \\
\hline total & & 12750918.4 & 100 \\
\hline
\end{tabular}


Determination of the optical purity of $3 \mathrm{~g}(97 \% \mathrm{ee})$.

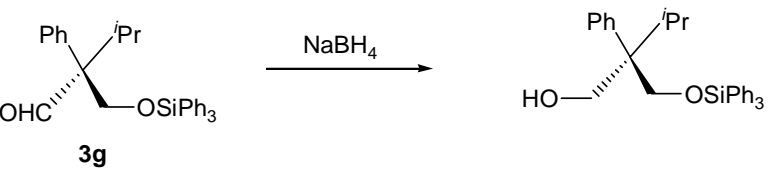

Chiral HPLC: Daicel CHIRALCEL OD-H, 500:0.2 Hexane:2-Propanol, 0.4 mL/min, $220 \mathrm{~nm}$ racemic

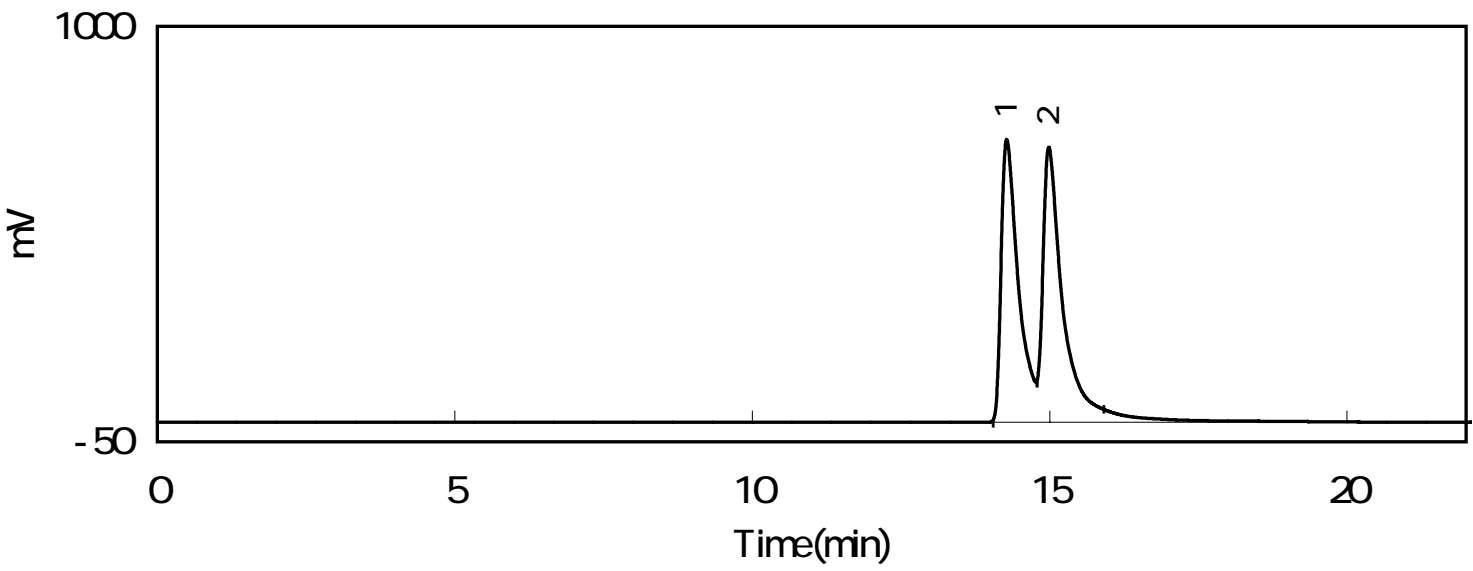

\begin{tabular}{|c|c|c|c|}
\hline \begin{tabular}{|l} 
No. \\
\end{tabular} & $\mathrm{Rt}(\mathrm{min})$ & Area & Area Percent \\
\hline 1 & 14.28 & 11930335.6 & 50.8026 \\
\hline 2 & 14.99 & 11553359 & 49.1974 \\
\hline total & & 23483694.6 & 100 \\
\hline
\end{tabular}

$97 \% e e$

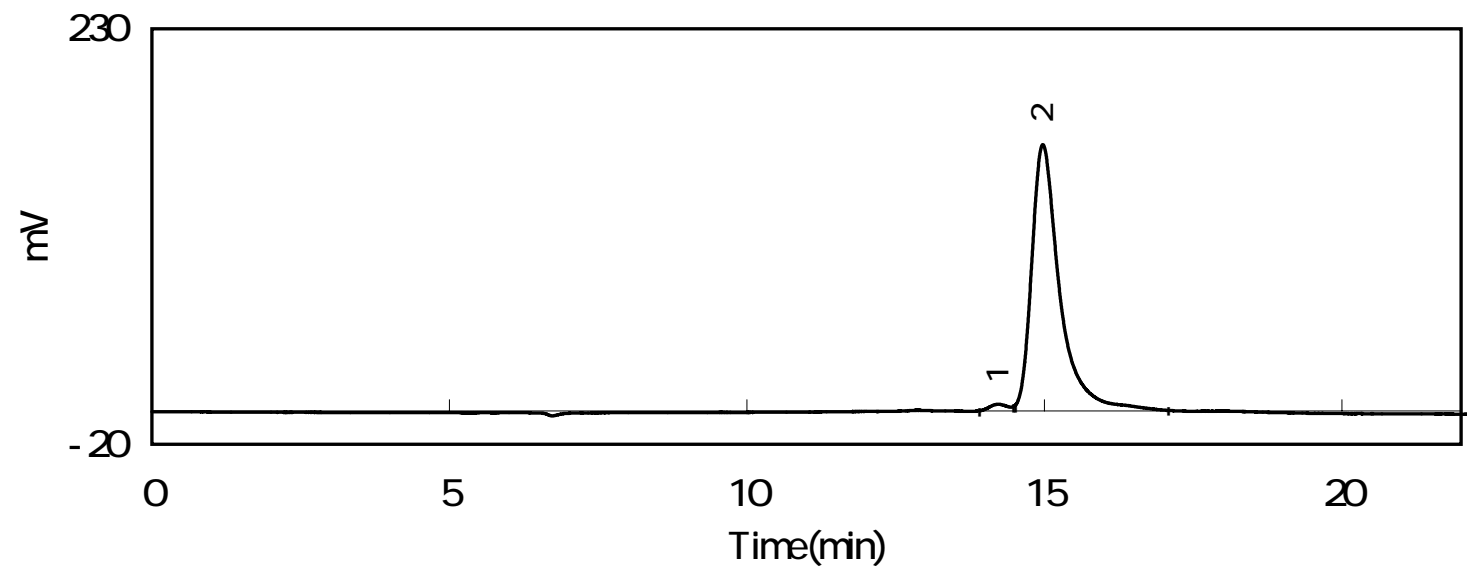

\begin{tabular}{|c|c|c|c|}
\hline No. & Rt (min) & Area & Area Percent \\
\hline$\overline{1}$ & 14.22 & 67404.8 & 1.3529 \\
\hline 2 & 14.97 & 4914853.2 & 98.6471 \\
\hline total & & 4982258 & 100 \\
\hline
\end{tabular}

